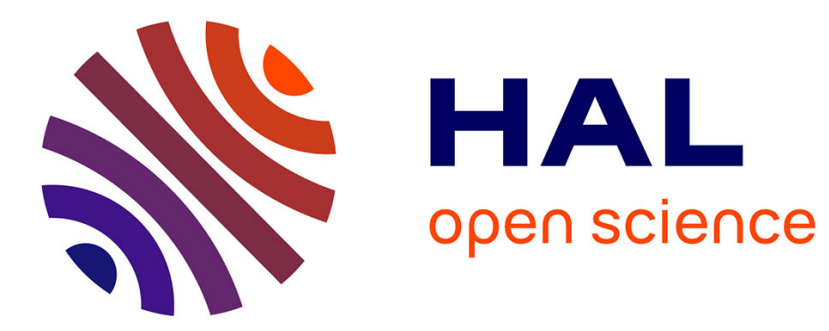

\title{
Wave turbulence in rapidly rotating flows
}

Fabien Bellet, Fabien Godeferd, Julian Scott, Claude Cambon

\section{To cite this version:}

Fabien Bellet, Fabien Godeferd, Julian Scott, Claude Cambon. Wave turbulence in rapidly rotating flows. Journal of Fluid Mechanics, 2006, 562, pp.83-121. 10.1017/S0022112006000929 . hal00274913

\section{HAL Id: hal-00274913 \\ https://hal.science/hal-00274913}

Submitted on 4 Oct 2011

HAL is a multi-disciplinary open access archive for the deposit and dissemination of scientific research documents, whether they are published or not. The documents may come from teaching and research institutions in France or abroad, or from public or private research centers.
L'archive ouverte pluridisciplinaire HAL, est destinée au dépôt et à la diffusion de documents scientifiques de niveau recherche, publiés ou non, émanant des établissements d'enseignement et de recherche français ou étrangers, des laboratoires publics ou privés. 


\title{
Wave-turbulence in rapidly rotating flows
}

\author{
By F. Bellet, F. S. Godeferd, J. F. Scott AND C. Cambon $\dagger$ \\ Laboratoire de Mécanique des Fluides et d'Acoustique UMR 5509 \\ École Centrale de Lyon, France
}

(Received 23 January 2006)

An Asymptotic Quasi-Normal Markovian (AQNM) model is developed in the limit of small Rossby number $R o$ and high Reynolds number, i.e. for rapidly rotating turbulent flow. Based on the 'slow' amplitudes of inertial waves, the kinetic equations are close to those that could be derived from Eulerian wave-turbulence theory. However, for their derivation we start from an EDQNM statistical closure model in which the velocity field is expanded in terms of the eigenmodes of the linear wave-regime. Unlike most wave-turbulence studies, our model accounts for the detailed anisotropy as the angular dependence in Fourier space. Nonlinear equations at small Rossby number are derived for the set $e, Z, h$ - energy, polarisation anisotropy, helicity —of spectral quantities which characterise second order two-point statistics in anisotropic turbulence, and which generate every quadratic moment of inertial wave amplitudes. In the simplest symmetry consistent with background equations, i.e. axisymmetry without mirror symmetry, $e, Z$ and $h$ depend on both the wave-vector modulus $k$ and its orientation $\theta$ to the rotation axis. We put the emphasis on obtaining accurate numerical simulations of a generalised Lin equation for the angular-dependent energy spectrum $e(k, \theta, t)$, in which the energy transfer reduces to integrals over surfaces given by the triadic resonant conditions of inertial waves. Starting from a pure three-dimensional isotropic state in which $e$ depends only on $k$ and $Z=h=0$, the spectrum develops an inertial range in the usual fashion 
as well as angular anisotropy. After the development phase, we observe the following features:

(a) a $k^{-3}$ power law for the spherically averaged energy spectrum. However, this is the average of power laws whose exponent varies with the direction of the wavevector from $k^{-2}$ for wavevectors near the plane perpendicular to the rotation axis, to $k^{-4}$ for parallel wavevectors.

(b) The spectral evolution is self-similar. This excludes the possibility of a purely twodimensional large-time limit.

(c) The energy density is very large near the perpendicular wavevector plane, but this singularity is integrable. As a result, the total energy has contributions from all directions and is not dominated by this singular contribution.

(d) The kinetic energy decays as $t^{-0.8}$, an exponent which is about half the one without rotation.

\section{Introduction}

Rotation of the reference frame is an important factor in some mechanisms of flow instability, and the study of rotating flows is interesting from the point of view of turbulence modelling in fields as diverse as engineering (e.g. turbomachinery and reciprocating engines with swirl and tumble), geophysics and astrophysics. Effects of mean curvature or of advection by a large eddy can be tackled using similar approaches.

Excluding altogether mean velocity and temperature gradients in the rotating frame of reference, the role of the Coriolis forces is still subtle and difficult to model, since the corresponding linear dynamics is made of oscillating motions, from the presence of neutral dispersive waves. In contrast with shear flows, there is no direct production of energy 
by linear effects, and the alteration of the distribution of energy is mainly controlled by nonlinear interactions, such as resonantly interacting waves for instance. Given the complexity of this general problem, we restrict our study to the case of homogeneous turbulence, assuming unbounded flows.

From several experimental, theoretical and numerical studies, in which rotation is suddenly applied to decaying homogeneous turbulence, some generally accepted statements are summarised as follows (Bardina et al., 1985, Cambon \& Jacquin, 1989, Jacquin et al., 1990, Cambon et al., 1992, Cambon et al., 1997). $\dagger$

- Rotation inhibits the energy cascade, so that the dissipation rate is reduced.

- The initial three-dimensional isotropy is broken through nonlinear interactions modified by rotation, so that anisotropy develops. This anisotropy may be characterized for instance by the angular distribution of energy in spectral space.

- If turbulence is initially anisotropic, the 'rapid' effects of rotation are short timescale linear dynamics which may be tackled as in Rapid Distortion Theory. They conserve the directional anisotropy and damp polarization anisotropy, resulting in a spectacular change of their relative contributions to the Reynolds Stress Tensor anisotropy.

Although the energy density associated with the plane of wavevectors perpendicular to the rotation axis can become very large due to the angular transfer, this does not necessarily mean that the flow approaches a two-dimensional state, in the sense that the overall energy is dominated by wavevectors near the plane. A careful application of the Taylor-Proudman theorem only permits to show that the 'slow manifold' is the twodimensional manifold at small Rossby number. But it does not imply the transition from three-dimensional to two-dimensional turbulence, which is a nonlinear transfer mechanism of energy from all the modes towards the two-dimensional ones; in other words $\dagger$ Other recent DNS and LES studies are rediscussed at the end. 
from 'rapid' to 'slow' ones. Two-dimensionalisation can be predicted using the Proudman theorem under two conditions: small non linearity and slow motion. The first condition is fulfilled at small Rossby number but not necessarily the second. In physical space, the slow, two-dimensional, manifold is the vertically averaged velocity field, such that $\partial / \partial z=0$, whereas in Fourier space it is the wave plane normal to the rotation axis. Results illustrating the mathematical subtleties arising from rapid rotation and dependence on the type of initial conditions can be found in Babin et al., 1997, Babin et al., 1999, Babin et al., 2001.

Two-point statistical closure (TPC) models have been extensively exploited to predict the nonlinear interactions, with satisfactorily good quantitative comparisons with DNS (Cambon et al., 1997, Godeferd \& Cambon, 1994, Godeferd \& Staquet, 2000). The mathematical formalism used in these previous studies has shown that it is fruitful to expand the fluctuating velocity field using the eigenmodes of the linear operator associated with the waves, as well as for analysing the nonlinear interactions in terms thereof. Similar eigenmodes decompositions (helical modes) were used by Cambon \& Jacquin, 1989, Waleffe, 1993, Smith \& Waleffe, 1999 and Morinishi et al., 2001a. Anisotropic EDQNM models in terms of helical modes will therefore be discussed to some extent in section 2 .

As regards wave-turbulence (WT) theory which was already studied long ago (see e.g. Benney \& Saffman, 1966), recent mathematical developments have renewed interest in flows which consist of superimposed dispersive waves, in which nonlinear interactions drive the long time behaviour(Caillol \& Zeitlin, 2000, Lvov \& Tabak, 2001, Galtier, 2003, Galtier et al., 2000). The link between the velocity $\boldsymbol{u}$ of a wave, given its wavevector $\boldsymbol{k}$, and its amplitude $\boldsymbol{a}$ can be established as

$$
\boldsymbol{u}(\boldsymbol{x}, t)=\boldsymbol{a}(t) \exp [\mathrm{i}(\boldsymbol{k} \cdot \boldsymbol{x}-\sigma t)]
$$

provided the analytical dispersion law $\sigma= \pm \sigma_{k}$ is known (Greenspan, 1990). The non- 
linear equations obtained from WT and TPC for the averaged amplitudes appear to be very similar, as briefly discussed hereafter.

The statistical homogeneity and quasi-normal assumptions used in TPC have equivalent counterparts in wave turbulence, obtained by assuming a priori random phases for the wave field. $\dagger$ The corresponding isotropic version of the quasi-normal assumption is discussed in Staquet \& Sommeria, 2002. Moreover, isotropic dispersion laws such that $\sigma_{k}=|\boldsymbol{k}|^{\alpha}$ in (1.1) are almost exclusively treated in WT for deriving Kolmogorov spectra, with the key hypothesis of constant isotropic energy fluxes across different scales associated with a wavenumber $|\boldsymbol{k}|$ (Zakharov et al., 1992). This contrasts with geophysical flows, in which dispersion laws are anisotropic: $\sigma_{k}=\beta k_{x} / k^{2}$ in the case of Rossby waves, $\sigma_{k}=2 \Omega k_{\|} / k$ for inertial waves, $\sigma_{k}=N k_{\perp} / k$ for internal gravity waves. $k_{x}, k_{\|}$and $k_{\perp}$ are the wavevector components respectively in the zonal direction and the directions parallel or perpendicular to the rotation/gravity axes. In the latter two three-dimensional cases, anisotropy manifests itself in the conical shape of iso-phase surfaces in experiments with localised forcing. Vertical plane cuts of these surfaces yield a 'St-Andrew cross' pattern, as observed in the experiments by McEwan, 1970, Mowbray \& Rarity, 1967, and numerically reproduced by Godeferd \& Lollini, 1999. Nonlinear interactions between waves also reflect anisotropy in the angular-dependent energy drain.

When considering Eulerian correlations, TPC and WT theories share a wide common background, seldom commented in the literature. Both WT and homogeneous TPC provide equations for the slow evolution of the waves' mean spectral energy densities. The energy transfer terms are cubic in terms of the wave amplitude, from triadic interac-

$\dagger$ The random phase approximation is commonly used by physicists. On the other hand, a mathematical justification for the Quasi-Normal relationship can be established in the weakly nonlinear limit of Wave Turbulence, following Benney \& Newell, 1967. 
tions. In WT only does one consider fourth-order transfer terms, from quartet interactions, when triple resonance is explicitly prevented by the dispersion law itself, or by geometrical constraints. This occurs for instance in shallow water waves. When triple resonances exist, as in rotating, stably stratified and MHD turbulence (Caillol \& Zeitlin, 2000, Galtier et al., 2000), WT kinetic equations have exactly the same structure as their counterpart in anisotropic TPC. Hence, WT and TPC have a common limit at very small interaction parameter, e.g. Rossby number Ro, Froude number $\mathrm{Fr}$, magnetic Reynolds number in MHD. We shall show that the precise form of the eddy damping parameter, which remains the heuristic correction to quasi-normal transfer in EDQNM, is unimportant in this limit. Its only role is to regularise the resonance operators. Beyond the weak nonlinearity assumption, the eddy damping, or more generally the nonlinear contribution to Kraichnan's response function (Kraichnan, 1958), can regain some importance for moderate interaction parameters, in allowing extrapolation from WT through TPC towards a larger domain, until the case of strong interactions is reached (e.g. pure isotropic turbulence without external or wave effects, for which classic multi-point closure models (MPC) work satisfactorily).

The paper is organised as follows. A survey of previous EDQNM models based on the helical modes decomposition is given in section 2, with their link to a rapid-slow analysis. Kinetic equations in the asymptotic limit of small Rossby number are derived for $e, Z, h$ in section 3 . The numerical method is presented in section 4 , numerical results are given in section 5, with recapitulation, discussions and perspectives in section 6 . A conclusion is proposed in section 7 . Tensorial details and tedious algebra are reported in appendices A to D. Appendix E provides details of the numerical procedure. 


\section{EDQNM in terms of the eigenmodes of inertial wave motion}

\subsection{The helical modes decomposition}

Rotating turbulence can be related to studies of incompressible homogeneous turbulence in the presence of a mean flow with space-uniform velocity gradients (Craya, 1958, Cambon \& Scott, 1999), provided a pure antisymmetric form $\epsilon_{i k j} \Omega_{k}$ be chosen, with $\Omega$ the angular velocity. It is nevertheless simpler to work with a coordinate system and velocity vectors in the rotating frame. In this non-inertial frame, rotation induces inertial centrifugal and Coriolis forces. Since the former can be incorporated in the pressure term, only the latter has to be taken into account in the following Navier-Stokes equations in the rotating frame:

$$
\begin{gathered}
\left(\partial_{t}+\boldsymbol{u} \cdot \boldsymbol{\nabla}\right) \boldsymbol{u}+2 \Omega \boldsymbol{n} \times \boldsymbol{u}+\nabla p-\nu \nabla^{2} \boldsymbol{u}=0 \\
\boldsymbol{\nabla} \cdot \boldsymbol{u}=0
\end{gathered}
$$

for the fluctuating velocity $\boldsymbol{u}$ and the pressure $p$ divided by density. The unit vector $\boldsymbol{n}$ denotes the direction aligned with the angular velocity of the rotating frame $\boldsymbol{\Omega}=\Omega \boldsymbol{n}$. Without loss of generality the fixed frame of reference is chosen such that $n_{i}=\delta_{i 3}$. Therefore $u_{3}$ is the axial velocity component.

In the inviscid linear regime, equations (2.1) becomes

$$
\frac{\partial \boldsymbol{u}}{\partial t}+2 \Omega \times \boldsymbol{u}+\nabla p=0
$$

Since the Coriolis force is not divergence-free, the pressure term has a nontrivial contribution to maintain the incompressibility constraint (2.2). The velocity can be eliminated between the latter equation and the Poisson equation for the pressure, for which a closed form is found

$$
\partial_{t}^{2}\left(\nabla^{2} p\right)+4 \Omega^{2} \nabla_{\|}^{2} p=0
$$


The reduced Laplacian operator along the axis of rotation is $\nabla_{\|}^{2}$. Although the primitive Poisson equation $\nabla^{2} p=f$ is parabolic, equation (2.4) is hyperbolic and admits propagating waves solutions. Interesting properties of these inertial waves are illustrated by cross shaped visualizations in the experiment by McEwan, 1970. Seeking plane waves solutions of (2.4) such that $p \propto e^{\mathrm{i}(\boldsymbol{k} \cdot \boldsymbol{x}-\sigma t)}$, one finds the dispersion law of inertial waves

$$
\sigma= \pm \sigma_{k}, \quad \sigma_{k}=2 \Omega \frac{k_{\|}}{k}=2 \Omega \cos \theta
$$

where $\theta$ is the angle between $\boldsymbol{k}$ and the rotation vector $\boldsymbol{\Omega}$. Without pressure, only the horizontal part of the flow is affected by circular periodic motion at constant frequency $2 \Omega$, but propagating waves cannot occur. Hence the fluctuating pressure is responsible both for anisotropic dispersivity and for horizontal-vertical coupling, coming from the divergence-free condition.

An equation similar to (2.4) is found for the vertical velocity component, and more generally for both the poloidal and toroidal potentials, detailed in appendix A. Without local forcing and boundary conditions, the linear problem can be very nicely recast in Fourier space as

$$
\frac{\partial \hat{u}_{i}}{\partial t}+2 \Omega P_{i n} \epsilon_{n 3 j} \hat{u}_{j}=0
$$

for the velocity Fourier coefficient $\hat{\boldsymbol{u}}(\boldsymbol{k}, t)$. The projection tensor is $P_{i n}=\delta_{i n}-k_{i} k_{n} / k^{2}$, $\delta_{i n}$ the Kronecker tensor, and $\epsilon_{i j k}$ the alternating tensor. In the turbulence community, (2.6) is known as the rapid distortion theory equation (RDT, as a reminder of the rapid-slow time scale separation implied when linearizing).

Given the incompressibility constraint $\hat{\boldsymbol{u}} \cdot \boldsymbol{k}=0$, it is easier to project the equation in the plane orthogonal to $\boldsymbol{k}$, using the frame $\left(\boldsymbol{e}^{(1)}, \boldsymbol{e}^{(2)}\right)$ (see the definition A 3 of these vectors in appendix A). The linear solution consists of a rotation of the initial Fourier component $\hat{\boldsymbol{u}}(\boldsymbol{k}, 0)$ about the axis $\boldsymbol{k}$ by an angle $\left(2 \Omega k_{\|} / k\right) t=\sigma_{k} t$. A tractable diagonal form of 
the corresponding Green's function is found in terms of the two complex eigenvectors $\boldsymbol{N}=\boldsymbol{e}^{(2)}-\mathrm{i} \boldsymbol{e}^{(1)}$ and $\boldsymbol{N}^{*}=\boldsymbol{N}(-\boldsymbol{k})=\boldsymbol{e}^{(2)}+\mathrm{i} \boldsymbol{e}^{(1)}$ in the plane normal to $\boldsymbol{k}$, namely

$$
G_{i j}^{R D T}\left(\boldsymbol{k}, t, t^{\prime}\right)=\frac{1}{2} \sum_{s= \pm 1} N_{i}(s \boldsymbol{k}) N_{j}(-s \boldsymbol{k}) e^{\mathrm{i} s \sigma_{k}\left(t-t^{\prime}\right)}
$$

which generates the linear solutions as

$$
\hat{u}_{i}(\boldsymbol{k}, t)=G_{i j}^{R D T}\left(\boldsymbol{k}, t, t^{\prime}\right) \hat{u}_{j}\left(\boldsymbol{k}, t^{\prime}\right) .
$$

These vectors have been used by different authors for two decades (Cambon \& Jacquin, 1989), and are called here helical modes after Waleffe, 1993. $\boldsymbol{N}$ and $\boldsymbol{N}^{*}$ prove useful in the pure rotation context since they provide a diagonal decomposition, for they are the eigenmodes of the curl operator, and therefore form a complete basis on which to project the Navier-Stokes equations. The resulting equations appear to be more tractable for discussing both linear and nonlinear operators, as well as for designing closure theories for turbulence, be it with rotation or not. Upon defining the velocity amplitudes $\xi_{1}, \xi_{-1}$ along $\boldsymbol{N}$ and $\boldsymbol{N}^{*}$ such that

$$
\hat{\boldsymbol{u}}(\boldsymbol{k}, t)=\xi_{+}(\boldsymbol{k}, t) \boldsymbol{N}(\boldsymbol{k})+\xi_{-}(\boldsymbol{k}, t) \boldsymbol{N}(-\boldsymbol{k}),
$$

the linear inviscid solution is

$$
\xi_{s}(\boldsymbol{k}, t)=\xi_{s}(\boldsymbol{k}, 0) \exp \left(2 \mathrm{i} s \Omega t \frac{k_{\|}}{k}\right), \quad s= \pm 1
$$

In terms of these modes, the complete nonlinear equation becomes

$$
\left(\frac{\partial}{\partial t}+\nu k^{2}-\mathrm{i} s\left(2 \Omega \frac{k_{\|}}{k}\right)\right) \xi_{s}=\sum_{s^{\prime}, s^{\prime \prime}= \pm 1} \int_{\boldsymbol{k}+\boldsymbol{p}+\boldsymbol{q}=\mathbf{0}} m_{s s^{\prime} s^{\prime \prime}}(\boldsymbol{k}, \boldsymbol{p}) \xi_{s^{\prime}}^{*}(\boldsymbol{p}, t) \xi_{s^{\prime \prime}}^{*}(\boldsymbol{q}, t) \mathrm{d}^{3} \boldsymbol{p}
$$

in which the l.h.s. linear operator is diagonal, and the r.h.s. is the modified form of the quadratic nonlinear term, exhibiting the interaction operator $\mathbf{m}$. This way, one may separate the rapid oscillating part of the complete nonlinear solution of (2.10) from slowly varying amplitudes $a_{s}, s= \pm 1$, as in multiple timescale analysis. The solution is formally 
written as

$$
\xi_{s}(\boldsymbol{k}, t)=a_{s}(\boldsymbol{k}, t) \exp \left(2 \mathrm{i} s \Omega t \frac{k_{\|}}{k}\right), \quad s= \pm 1
$$

and from (2.8) the $i$-th component of the Fourier coefficient of the velocity is

$$
\hat{u}_{i}(\boldsymbol{k}, t)=\sum_{s= \pm 1} a_{s}(\boldsymbol{k}, t) \exp \left(\mathrm{i} s \sigma_{k} t\right) N_{i}(s \boldsymbol{k})
$$

In the evolution equation for the slow amplitudes $a_{s}$, the linear operators are absorbed in the nonlinear one, as integrating factors:

$\dot{a}_{s}+\nu k^{2} a_{s}=\sum_{s^{\prime}, s^{\prime \prime}= \pm 1} \int_{\boldsymbol{k}+\boldsymbol{p}+\boldsymbol{q}=\mathbf{0}} \exp \left(-2 \mathrm{i} \Omega\left(s \frac{k_{\|}}{k}+s^{\prime} \frac{p_{\|}}{p}+s^{\prime \prime} \frac{q_{\|}}{q}\right) t\right) m_{s s^{\prime} s^{\prime \prime}}(\boldsymbol{k}, \boldsymbol{p}) a_{s^{\prime}}^{*}(\boldsymbol{p}, t) a_{s^{\prime \prime}}^{*}(\boldsymbol{q}, t) \mathrm{d}^{3} \boldsymbol{p}$

with the influence matrix $m_{s s^{\prime} s^{\prime \prime}}$ given in Cambon et al., 1997, Waleffe, 1993. Equation (2.13) demonstrates the importance of the resonant triads $\sigma_{k} \pm \sigma_{p} \pm \sigma_{q}=0$ that appear when the phase term in (2.13) is zero:

$$
\frac{k_{\|}}{k} \pm \frac{p_{\|}}{p} \pm \frac{q_{\|}}{q}=0 \quad \text { with } \quad \boldsymbol{k}+\boldsymbol{p}+\boldsymbol{q}=0
$$

Resonant and almost resonant triads are expected to dominate nonlinear slow motion, since significant non zero values of $k_{\|} / k \pm p_{\|} / p \pm q_{\|} / q$ in the r.h.s. of (2.13) severely damp the nonlinearity by scrambling. In that case, why not obtain a simplified model by solving equation (2.13) with an integral restricted to the resonant triads? Because the resonant surfaces are complex enough for very accurate interpolation to be needed, rendering the resulting computation only relevant for a smooth distribution of the slow amplitudes $a_{s}$ in Fourier space. Such a smooth distribution cannot represent turbulence, so that one has to resort to describing statistical quantities instead, which are naturally smooth. Closing equations for these statistical moments renders a quasi-normal assumption necessary, yet resonant surface integrals can be employed, as described in the following section. 


\subsection{Equations for second order correlations}

From the definition of the second order spectral tensor,

$$
<\hat{u}_{i}(\boldsymbol{k}, t) \hat{u}_{j}^{*}(\boldsymbol{p}, t)>=\Phi_{i j}(\boldsymbol{k}, t) \delta(\boldsymbol{k}-\boldsymbol{p})
$$

and the relation (2.12) which links the fluctuating velocity and the slow-varying amplitudes, one may obtain the correlation tensor

$$
\Phi_{i j}=\sum_{s, s^{\prime}= \pm 1} A_{s s^{\prime}}(\boldsymbol{k}, t) N_{i}(s \boldsymbol{k}) N_{j}\left(s^{\prime} \boldsymbol{k}\right) e^{\mathrm{i}\left(s+s^{\prime}\right) \sigma t}
$$

where the $A_{s s^{\prime}}$ are second order correlations of the slow amplitudes

$$
<a_{s}(\boldsymbol{k}, t) a_{-s^{\prime}}\left(\boldsymbol{k}^{\prime}, t\right)>=A_{s s^{\prime}}(\boldsymbol{k}, t) \delta\left(\boldsymbol{k}+\boldsymbol{k}^{\prime}\right) .
$$

These correlations are closely related to the set $e, \zeta, h$ (energy, polarisation anisotropy, helicity, see appendix B), as follows:

$$
e=A_{1 ;-1}+A_{-1 ; 1}, \quad \zeta=2 A_{-1 ;-1} e^{-2 \mathrm{i} \sigma t}, \quad h=A_{-1 ; 1}-A_{1 ;-1} .
$$

The following system of equations is obtained for the second order spectral tensor

$$
\begin{aligned}
\left(\frac{\partial}{\partial t}+2 \nu k^{2}\right) e & =T^{(e)}=\frac{1}{2}\left(\tau_{i i}+\tau_{i i}^{*}\right) \\
\left(\frac{\partial}{\partial t}+2 \nu k^{2}+2 \mathrm{i} \sigma_{k}\right) \zeta & =T^{(\zeta)}=\frac{1}{2} N_{i} N_{j}\left(\tau_{i j}+\tau_{j i}^{*}\right) \\
\left(\frac{\partial}{\partial t}+2 \nu k^{2}\right) h & =T^{(h)}=-\frac{1}{2} \mathrm{i} \frac{k_{l}}{k} \varepsilon_{l i j}\left(\tau_{i j}+\tau_{j i}^{*}\right)
\end{aligned}
$$

which may also be written for the $A_{s s^{\prime}}$ :

$$
\frac{\partial A_{s s^{\prime}}}{\partial t}=\frac{1}{4} N_{i}^{*}(s \boldsymbol{k}) N_{j}^{*}\left(s^{\prime} \boldsymbol{k}\right)\left(\tau_{i j}+\tau_{j i}^{*}\right) e^{-\mathrm{i}\left(s+s^{\prime}\right) \sigma t} .
$$

Contributions from velocity triple correlations are gathered into the symmetric generalized spectral transfer tensor $\tau_{i j}+\tau_{j i}^{*}$, which is an unclosed term in the equation that governs $\Phi_{i j}$.

If the system of equations (2.19)-(2.21) is started with three-dimensional isotropic initial data, that is with $e(k, t=0)=E(k) /\left(4 \pi k^{2}\right)$ and $\zeta=h=0, E(k)$ being a 
given distribution of spectral energy, anisotropy which reflects the transition towards two-dimensional structure may be created only by the nonlinear spectral transfer terms. This anisotropy has to be consistent with the axisymmetry of the problem, so that isotropy is broken through the dependence with $\theta=\cos ^{-1}\left(k_{\|} / k\right)$ of the spectral quantities $e=e(k, \theta, t)$ and $\zeta=\zeta(k, \theta, t)$. Note that $\zeta(\boldsymbol{k})$ has to be zero when $\boldsymbol{k}$ is parallel to the vertical axis, if the closure model is to agree with the symmetries of the rotating NavierStokes equations (axisymmetry without mirror symmetry is ensured by the equations if initially verified).

\subsection{Improved EDQNM modelling from previous versions}

The derivation of a general quasi-normal (QN) model using the Green's function of the linear operator is detailed in Cambon \& Scott, 1999, and references therein. The corresponding expression for the tensor $\tau_{i j}$, which reflects the contribution of triple correlations to the equation governing $\Phi_{i j}$, consists of an integral over the third-order spectral tensor, whose equation is closed by the quasi-normal relationship as follows:

$$
\begin{aligned}
\tau_{i j}(\boldsymbol{k}, t)= & P_{j k l}(\boldsymbol{k}) \int_{-\infty}^{t} \int_{\boldsymbol{k + p + q}=\mathbf{0}} G_{i m}^{(0)}\left(\boldsymbol{k}, t, t^{\prime}\right) G_{k p}^{(0)}\left(\boldsymbol{p}, t, t^{\prime}\right) G_{l q}^{(0)}\left(\boldsymbol{q}, t, t^{\prime}\right) \\
& \Phi_{p n}\left(\boldsymbol{p}, t^{\prime}\right)\left[\frac{1}{2} P_{m n r}(\boldsymbol{k}) \Phi_{q r}\left(\boldsymbol{q}, t^{\prime}\right)+P_{q n r}(\boldsymbol{q}) \Phi_{m r}\left(\boldsymbol{k}, t^{\prime}\right)\right] \mathrm{d}^{3} \boldsymbol{p} \mathrm{d} t^{\prime}
\end{aligned}
$$

in which $P_{i j k}(\boldsymbol{k})=k_{j} P_{i k}(\boldsymbol{k})+k_{k} P_{i j}(\boldsymbol{k})$ and the viscous term is reinserted at no cost in the RDT Green's function as

$$
G_{i j}^{(0)}\left(\boldsymbol{k}, t, t^{\prime}\right)=G_{i j}^{R D T}\left(\boldsymbol{k}, t, t^{\prime}\right) e^{-\nu k^{2}\left(t-t^{\prime}\right)} .
$$

$\mathbf{G}^{(0)}$ directly generates the zeroth-order Kraichnan response function, which is a key quantity in any Renormalized Perturbation Theory. The triple product of Green's functions in (2.23) arises from the Green's function solution of the third-order moments equation, and integration is performed over the triads such that $\boldsymbol{k}+\boldsymbol{p}+\boldsymbol{q}=\mathbf{0}$ so that $\boldsymbol{q}$ should be replaced by $-\boldsymbol{k}-\boldsymbol{p}$ throughout the integrand. The only assumption in (2.23) is the 
quasi-normal writing of fourth-order velocity correlations in terms of products of secondorder ones. The difference with quasi-isotropic versions is that the exact linear terms are taken into account, and anisotropy is explicitly retained in the formulation.

As in all Renormalized Perturbation Theory, which were developed in isotropic turbulence, it is necessary to replace the 'bare' viscous Green's function (2.24) by a renormalized version, otherwise the resulting model is not realizable (Orszag, 1970).

This is done in the eddy damped quasi-normal model (EDQN) by changing the viscous factor $\nu k^{2}$ into $\mu=\nu k^{2}+\mu^{\prime}$ in the zeroth-order response function (2.24). Accordingly, the EDQN nonlinear transfer term is

$$
\begin{aligned}
\tau_{i j}(\boldsymbol{k}, t) & =P_{j k l}(\boldsymbol{k}) \int_{-\infty}^{t} \int_{\boldsymbol{k}+\boldsymbol{p}+\boldsymbol{q}=\mathbf{0}} G_{i m}\left(\boldsymbol{k}, t, t^{\prime}\right) G_{k p}\left(\boldsymbol{p}, t, t^{\prime}\right) G_{l q}\left(\boldsymbol{q}, t, t^{\prime}\right) \\
& \Phi_{p n}\left(\boldsymbol{p}, t^{\prime}\right)\left[\frac{1}{2} P_{m n r}(\boldsymbol{k}) \Phi_{q r}\left(\boldsymbol{q}, t^{\prime}\right)+P_{q n r}(\boldsymbol{q}) \Phi_{m r}\left(\boldsymbol{k}, t^{\prime}\right)\right] \mathrm{d}^{3} \boldsymbol{p} \mathrm{d} t^{\prime}
\end{aligned}
$$

with the mollified kernel

$$
G_{i j}\left(\boldsymbol{k}, t, t^{\prime}\right)=G_{i j}^{R D T}\left(\boldsymbol{k}, t, t^{\prime}\right) e^{-\nu k^{2}\left(t-t^{\prime}\right)} e^{-\int_{t^{\prime}}^{t} \mu^{\prime}\left(k, t^{\prime \prime}\right) \mathrm{d} t^{\prime \prime}}
$$

The regularisation coefficient $\mu^{\prime}(k, t)=A\left(\int_{0}^{k} p^{2} E(p, t) \mathrm{d} p\right)^{1 / 2}$ was proposed by Pouquet et al., 1975 for isotropic EDQNM. This eddy damping involves the inverse of the integral time-scale introduced by Comte-Bellot \& Corrsin, 1971 instead of the local estimate $\mu^{\prime} \sim k^{3 / 2} E^{1 / 2}$ initially proposed by Orszag, 1970.

Equation (2.25) exhibits the generic anisotropic structure of most generalised classical theories dealing with two-point closure, or renormalized perturbation theories, but its zeroth order limit involves, as the zeroth-order response tensor, a full RDT viscous Green's function which is more relevant than the laminar viscous one.

We now discuss the Markovianisation procedure, i.e. the way we treat time-dependency in the integrands that connect the transfer term to second order correlations. We notice three kinds of time-dependent terms in (2.25): 
(a) viscous or viscous-damping terms $\exp \left(\int_{t^{\prime}}^{t} \mu \mathrm{d} t^{\prime \prime}\right)$ which we write $V\left(t, t^{\prime}\right)$,

(b) components of the RDT Green's function $\mathbf{G}\left(t, t^{\prime}\right)$, proportional to $\exp \left( \pm \mathrm{i} \sigma\left(t-t^{\prime}\right)\right)$,

(c) products of the second-order spectral tensor components $\Phi_{. .}\left(t^{\prime}\right)$, or equivalent products of $e\left(t^{\prime}\right), \zeta\left(t^{\prime}\right)$ and $h\left(t^{\prime}\right)$.

In the Markovianisation procedure used in classical $\mathrm{EDQNM}_{1}, V\left(t, t^{\prime}\right)$ is assumed to be rapidly decreasing in terms of the time separation $t-t^{\prime}$ and the eddy-viscous damping $\mu$ varies slowly enough to be assumed constant. All the other terms are evaluated at $t^{\prime}=t$ and are consistently replaced by $\mathbf{G}(t, t)$ and $\mathbf{\Phi}(t)$. However, since $\mathbf{G}\left(t-t^{\prime}\right)$ is responsible for breaking the initial isotropy in rotating turbulence, this model does not produce any anisotropic structure.

This drawback is removed in $\mathrm{EDQNM}_{2}$ by approximating only the $e, \zeta$ and $h$ terms at $t=t^{\prime}$, and retaining the complete $V\left(t, t^{\prime}\right)$ and $G\left(t, t^{\prime}\right)$ terms in the time integrand. The latter integral of a three-fold product of response functions yields the following closed nonlinear transfer:

$$
T^{(e, Z, h)}=\sum_{s= \pm 1, s^{\prime}= \pm 1, s^{\prime \prime}= \pm 1} \int \frac{S^{s s^{\prime} s^{\prime \prime}}(e, \zeta, h)}{\mu_{k p q}+\mathrm{i}\left(s \sigma_{k}+s^{\prime} \sigma_{p}+s^{\prime \prime} \sigma_{q}\right)} \mathrm{d}^{3} \boldsymbol{p} .
$$

$\mathrm{EDQNM}_{2}$ compared well with high-resolution $528 \times 128 \times 128$ large eddy simulations (LES) in view of the creation of directional anisotropy, and for predicting the development of anisotropy (Cambon et al., 1997). However, regarding the rapid-slow decomposition (2.11), one can enlarge the scope of application of $\mathrm{EDQNM}_{2}$ by loosening the 'slow' approximation of $e, \zeta$ and $h$. In agreement with equation (2.18), only $Z=2 A_{-1 ;-1}$ has to be considered as a slow variable. In this $\mathrm{EDQNM}_{3}$ model, $e\left(t^{\prime}\right)=e(t), h\left(t^{\prime}\right)=h(t)$, $Z\left(t^{\prime}\right)=Z(t)$, but in the integral, $\zeta\left(t^{\prime}\right)=Z(t) \exp \left(-2 \mathrm{i} \sigma t^{\prime}\right)$; and $V\left(t, t^{\prime}\right), G\left(t, t^{\prime}\right)$ as before. This model consistently treats all $A_{s s^{\prime}}$ as slow variables. $\mathrm{EDQNM}_{3}$ only slightly differs from $\mathrm{EDQNM}_{2}$, but offers valuable advantages. First, it is exactly equivalent to the model derived directly from the slow amplitudes, using (2.11) and (2.13). As a consequence, its 
asymptotic limit at large $t$, and small Rossby number, when $\mu \ll 2 \Omega$, coincides exactly with Eulerian wave-turbulence theory. Second, realizability can be demonstrated in this limit (Bellet, 2003), while it is not mathematically ensured in the $\mathrm{EDQNM}_{2}$ version.

In $\mathrm{EDQNM}_{3}$ the transfer tensor becomes

$$
\begin{array}{r}
\tau_{i j}(\boldsymbol{k}, t)=\frac{1}{2} \sum_{s, s^{\prime}, s^{\prime \prime}, s_{1}, s_{2}} P_{j l m}(\boldsymbol{k}) \int_{\mathbb{R}^{3}} \int_{-\infty}^{t} e^{\left[\mathrm{i}\left(s \sigma_{k}+s^{\prime} \sigma_{p}+s^{\prime \prime} \sigma_{q}\right)-\mu_{k p q}\right]\left(t-t^{\prime}\right)} e^{\mathrm{i}\left(s^{\prime}+s_{1}\right) \sigma_{p} t^{\prime}} \times \\
N_{i}(s \boldsymbol{k}) N_{l}\left(s^{\prime} \boldsymbol{p}\right) N_{m}\left(s^{\prime \prime} \boldsymbol{q}\right) N_{k}\left(s_{1} \boldsymbol{p}\right) A_{s^{\prime} s_{1}}(\boldsymbol{p}, t) \times \\
{\left[e^{\mathrm{i}\left(s^{\prime \prime}+s_{2}\right) \sigma_{q} t^{\prime}} \frac{1}{2} P_{p k n}(\boldsymbol{k}) N_{p}(-s \boldsymbol{k}) N_{n}\left(s_{2} \boldsymbol{q}\right) A_{s^{\prime \prime} s_{2}}(\boldsymbol{q}, t)\right.} \\
\left.+e^{\mathrm{i}\left(s+s_{2}\right) \sigma_{k} t^{\prime}} P_{p k n}(\boldsymbol{q}) N_{p}\left(-s^{\prime \prime} \boldsymbol{q}\right) N_{n}\left(s_{2} \boldsymbol{k}\right) A_{s s_{2}}(\boldsymbol{k}, t)\right] \mathrm{d} t^{\prime} \mathrm{d}(\boldsymbol{p} .28)
\end{array}
$$

in which the quadratic slow amplitude terms have been set to their instantaneous values at $t$, and $\mu_{k p q}=\mu(k, t)+\mu(p, t)+\mu(q, t)$. Analytical time integration of exponential terms $e^{\mathrm{i}(. .) t^{\prime}}$ is performed assuming constant $\mu$, so that $\mathrm{EDQNM}_{3}$ finally is

$$
\begin{aligned}
\tau_{i j}(\boldsymbol{k}, t)=\frac{1}{2} \sum_{s, s^{\prime}, s^{\prime \prime}, s_{1}, s_{2}} P_{j l m}(\boldsymbol{k}) \int_{\mathbb{R}^{3}} N_{i}(s \boldsymbol{k}) N_{l}\left(s^{\prime} \boldsymbol{p}\right) N_{m}\left(s^{\prime \prime} \boldsymbol{q}\right) N_{k}\left(s_{1} \boldsymbol{p}\right) A_{s^{\prime} s_{1}}(\boldsymbol{p}, t) \times \\
e^{\mathrm{i}\left(s^{\prime}+s_{1}\right) \sigma_{p} t}\left[\frac{1}{2} P_{p k n}(\boldsymbol{k}) N_{p}(-s \boldsymbol{k}) N_{n}\left(s_{2} \boldsymbol{q}\right) \frac{A_{s^{\prime \prime} s_{2}}(\boldsymbol{q}, t) e^{\mathrm{i}\left(s^{\prime \prime}+s_{2}\right) \sigma_{q} t}}{\mu_{k p q}-\mathrm{i}\left(s \sigma_{k}-s_{1} \sigma_{p}-s_{2} \sigma_{q}\right)}\right. \\
\left.+P_{p k n}(\boldsymbol{q}) N_{p}\left(-s^{\prime \prime} \boldsymbol{q}\right) N_{n}\left(s_{2} \boldsymbol{k}\right) \frac{A_{s s_{2}}(\boldsymbol{k}, t) e^{\mathrm{i}\left(s+s_{2}\right) \sigma_{k} t}}{\mu_{k p q}-\mathrm{i}\left(s^{\prime \prime} \sigma_{q}-s_{1} \sigma_{p}-s_{2} \sigma_{k}\right)}\right] \mathrm{d}^{3} \boldsymbol{p}(2.29)
\end{aligned}
$$

The usual tensorial writing of the transfer is used in the above equation, but it is easy to derive corresponding equations for the sets $\left(A_{1 ;-1}, A_{-1 ; 1}, A_{-1 ;-1}\right)$ or $(e, Z, h)$, as in Cambon et al., 1997. The difference between $\mathrm{EDQNM}_{2}$ and $\mathrm{EDQNM}_{3}$ only deals with the rapid Coriolis-induced phase of $\zeta$, or for $s^{\prime}=s_{1}$ and $s^{\prime \prime}=s_{2}$ terms, so that the final EDQNM $_{3}$ expressions for $\left(T^{e}, T^{Z}, T^{h}\right)$ are very close to those in Cambon et al., 1997 (also in the appendix of Cambon et al., 2004b, denoted CRG).

Another way of obtaining these closed equations for the slow amplitude double correlations is to build moment equations in terms of $\left\langle a_{s} a_{s^{\prime}}\right\rangle,\left\langle a_{s} a_{s^{\prime}} a_{s^{\prime \prime}}\right\rangle$, etc., directly from equations (2.13) and (2.17). Since the passage from $\hat{u}_{i}$ to $a_{s}$ involves the eigenmodes 
$N_{i}$, as do the Green functions and projection operators, the amount of work is equivalent.

The "Byzantine use of projectors" (Turner, 1999), is not an impediment to derive final equations in terms of slow amplitudes only, which is the aim of wave-turbulence Eulerian theory, as described in the following section.

\section{The Asymptotic Quasi-Normal Markovian Model}

\subsection{Removing rapid oscillations}

A simplified form of $\tau_{i j}$ is first obtained by taking $t$ large, dropping those terms in equation (2.29) which are rapidly oscillating as functions of $\boldsymbol{p}$. Upon close examination of the corresponding terms in $(2.29)$, this results in

$$
\tau_{i j}=R_{i j}+\sum_{s_{3}, s_{4}= \pm 1} I_{i j ; s_{3} s_{4}} A_{s_{3} s_{4}} e^{\mathrm{i}\left(s_{3}+s_{4}\right) \sigma_{k} t}
$$

where

$$
\begin{array}{r}
R_{i j}(\boldsymbol{k}, t)=\frac{1}{4} \sum_{s, s^{\prime}, s^{\prime \prime}} P_{j l m}(\boldsymbol{k}) P_{p k n}(\boldsymbol{k}) N_{i}(s \boldsymbol{k}) N_{p}(-s \boldsymbol{k}) \times \\
\int_{\mathbb{R}^{3}} N_{k}\left(-s^{\prime} \boldsymbol{p}\right) N_{l}\left(s^{\prime} \boldsymbol{p}\right) N_{m}\left(s^{\prime \prime} \boldsymbol{q}\right) N_{n}\left(-s^{\prime \prime} \boldsymbol{q}\right) \frac{A_{s^{\prime} ;-s^{\prime}}(\boldsymbol{p}, t) A_{s^{\prime \prime} ;-s^{\prime \prime}}(\boldsymbol{q}, t)}{\mu_{k p q}-\mathrm{i}\left[s \sigma(\boldsymbol{k})+s^{\prime} \sigma(\boldsymbol{p})+s^{\prime \prime} \sigma(\boldsymbol{q})\right]} \mathrm{d}^{3} \boldsymbol{p},
\end{array}
$$

which only involves the real components $A_{s ;-s}(\boldsymbol{k}, t)$ and

$$
\begin{array}{r}
I_{i j ; s_{3} s_{4}}=\frac{1}{2} \sum_{s^{\prime}, s^{\prime \prime}} P_{j l m}(\boldsymbol{k}) N_{i}\left(s_{3} \boldsymbol{k}\right) N_{n}\left(s_{4} \boldsymbol{k}\right) \times \\
\int_{\mathbb{R}^{3}} N_{k}\left(-s^{\prime} \boldsymbol{p}\right) N_{l}\left(s^{\prime} \boldsymbol{p}\right) N_{m}\left(s^{\prime \prime} \boldsymbol{q}\right) N_{p}\left(-s^{\prime \prime} \boldsymbol{q}\right) \frac{P_{p k n}(\boldsymbol{q}) A_{s^{\prime} ;-s^{\prime}}(\boldsymbol{p}, t)}{\mu_{k p q}-\mathrm{i}\left[s^{\prime} \sigma(\boldsymbol{p})+s^{\prime \prime} \sigma(\boldsymbol{q})-s_{4} \sigma(\boldsymbol{k})\right]} \mathrm{d}^{3} \boldsymbol{p} .
\end{array}
$$

Both $R_{i j}$ and $I_{i j ; s_{3} s_{4}}$ are slowly varying quantities, as is $A_{s_{1} s_{2}}$, so the above expression for $\tau_{i j}$ explicitly shows the fast-time dependency. Substituting the asymptotic result for $\tau_{i j}$ into the evolution equation for $A_{s s^{\prime}}$ and dropping those terms which are rapidly oscillating in time, so that only terms with $s_{3}+s_{4}-s-s^{\prime}=0$ are conserved in equation (2.22), 
yields the equation

$$
\frac{\partial A_{s ;-s}}{\partial t}=B_{s} A_{s ;-s}+C_{s}
$$

with

$$
\begin{gathered}
B_{s}(\boldsymbol{k}, t)=-2 \sum_{s^{\prime} s^{\prime \prime}} \int_{\mathbb{R}^{3}} g_{s^{\prime} s^{\prime \prime}} \frac{\mu_{k p q}}{\mu_{k p q}^{2}+F_{s^{\prime} s^{\prime \prime}}^{2}} A_{s s^{\prime}}(\boldsymbol{p}, t) \mathrm{d}^{3} \boldsymbol{p} \\
C_{s}(\boldsymbol{k}, t)=2 \sum_{s^{\prime} s^{\prime \prime}} \int_{\mathbb{R}^{3}} \gamma_{s^{\prime} s^{\prime \prime}} \frac{\mu_{k p q}}{\mu_{k p q}^{2}+F_{s^{\prime} s^{\prime \prime}}^{2}} A_{s s^{\prime}}(\boldsymbol{p}, t) A_{s s^{\prime \prime}}(\boldsymbol{q}, t) \mathrm{d}^{3} \boldsymbol{p}
\end{gathered}
$$

and the equation

$$
\frac{\partial Z}{\partial t}=D Z, \quad Z=2 A_{-1 ;-1}
$$

with

$$
D(\boldsymbol{k}, t)=-\sum_{s^{\prime} s^{\prime \prime}} \int_{\mathbb{R}^{3}} g_{s^{\prime} s^{\prime \prime}} \frac{\mu_{k p q}+\mathrm{i} F_{s^{\prime} s^{\prime \prime}}}{\mu_{k p q}^{2}+F_{s^{\prime} s^{\prime \prime}}^{2}} e(\boldsymbol{p}, t) \mathrm{d}^{3} \boldsymbol{p}
$$

The expression of $\gamma_{s^{\prime} s^{\prime \prime}}$ is given in the next section in terms of $g_{s^{\prime} s^{\prime \prime}}$, itself detailed in appendix D in terms of $(k, p, q)$. Yet again, as in $\mathrm{EDQNM}_{2}$, dispersion frequencies appear only through the term $F_{s^{\prime} s^{\prime \prime}}=\sigma_{k}+s^{\prime} \sigma_{p}+s^{\prime \prime} \sigma_{q}$.

\subsection{The asymptotic limit of small damping}

The asymptotic evolution equations are obtained by taking the limit $\mu_{k p q} \rightarrow 0$ in the above expressions for $C_{s}, B_{s}$ and $D$. In wave-turbulence theory, it is usual to replace the volume integral by a surface integral over the resonant surface in this limit. $\dagger$ However, taking the limit is not simple, and is valid only for the real part of the rational fraction involved in equation (3.5) or in equations (3.3) and (3.4), but not for the imaginary part of the fraction appearing in the $D$ coefficient. In this section, we briefly present the path to the final surface integrals, taking the limits for granted. Details on their obtention are given in appendix $\mathrm{C}$.

$\dagger$ For instance, the Plemelj-Sokhotsky formula $\frac{1}{d-\mathrm{i} x}=\pi \delta(x)+\mathrm{i} \mathcal{P}\left(\frac{1}{x}\right)$ is used by Caillol \& Zeitlin, 2000, where $\mathcal{P}$ is the principal value. 
Starting with the real part, it is necessary to parameterize the resonant surface, which is the set

$$
S_{s^{\prime} s^{\prime \prime}}(\boldsymbol{k})=\left\{\boldsymbol{p} \in \mathbb{R}^{3}, \quad \text { such that } \sigma(\boldsymbol{k})+s^{\prime} \sigma(\boldsymbol{p})+s^{\prime \prime} \sigma(-\boldsymbol{k}-\boldsymbol{p})=0\right\} .
$$

We also introduce the parameter $d$ which plays the role of $\mu_{k p q}$ in the above. Knowing the following limit of the function of $x$

$$
f_{d}(x)=\frac{d}{d^{2}+x^{2}} \rightarrow \pi \delta(x) \quad \text { for } \quad d \rightarrow 0
$$

one finds that

$$
\int_{\mathbb{R}^{3}} A(\boldsymbol{k}, \boldsymbol{p}) \frac{d}{d^{2}+F_{s^{\prime} s^{\prime \prime}}^{2}} \mathrm{~d}^{3} \boldsymbol{p} \rightarrow \pi \int_{\mathbb{R}^{3}} \delta\left(F_{s^{\prime} s^{\prime \prime}}\right) A(\boldsymbol{k}, \boldsymbol{p}) \mathrm{d}^{3} \boldsymbol{p}
$$

when $d$ goes to zero. The latter integral is recast as the surface integral

$$
\int_{S_{s^{\prime} s^{\prime \prime}}} \frac{A(\boldsymbol{k}, \boldsymbol{p})}{\alpha_{s^{\prime} s^{\prime \prime}}} \mathrm{d} S
$$

in which $\alpha_{s^{\prime} s^{\prime \prime}}$ denotes the gradient of the function $F_{s^{\prime} s^{\prime \prime}} / \pi$ along the direction normal to the surface $F_{s^{\prime} s^{\prime \prime}}=0$ :

$$
\alpha_{s^{\prime} s^{\prime \prime}}=\frac{1}{\pi}\left|s^{\prime} \boldsymbol{C}_{g}(\boldsymbol{p})-s^{\prime \prime} \boldsymbol{C}_{g}(\boldsymbol{q})\right| .
$$

The coefficient $\alpha_{s^{\prime} s^{\prime \prime}}$ involves the group velocity $\boldsymbol{C}_{g}(\boldsymbol{k})=-\left(2 \Omega k_{\perp} / k^{2}\right) \boldsymbol{e}^{(2)}(\boldsymbol{k})$ and is therefore angular dependent.

For the imaginary part of the fraction involved in the equation (3.5) for $D$, one uses the limit at vanishing $d$ :

$$
\int_{\mathbb{R}^{3}} \frac{F_{s^{\prime} s^{\prime \prime}}}{d^{2}+F_{s^{\prime} s^{\prime \prime}}^{2}} A(\boldsymbol{k}, \boldsymbol{p}) \mathrm{d}^{3} \boldsymbol{p} \rightarrow f_{\mathbb{R}^{3}} \frac{A(\boldsymbol{k}, \boldsymbol{p})}{F_{s^{\prime} s^{\prime \prime}}} \mathrm{d}^{3} \boldsymbol{p}
$$

which is a principal value integral in the vicinity of the resonant surface.

Accordingly, the coefficients $B_{s}, C_{s}, D$ take the following asymptotic values, in the limit of vanishing $\mu_{k p q}$ :

$$
B_{s}(\boldsymbol{k}, t)=-2 \sum_{s^{\prime}, s^{\prime \prime}} \int_{S_{s^{\prime} s^{\prime \prime}}} \frac{g_{s^{\prime} s^{\prime \prime}}}{\alpha_{s^{\prime} s^{\prime \prime}}} A_{s s^{\prime}}(\boldsymbol{p}, t) \mathrm{d} S
$$




$$
\begin{gathered}
\text { Wave-turbulence in rapidly rotating flows } \\
C_{s}(\boldsymbol{k}, t)=2 \sum_{s^{\prime} s^{\prime \prime}} \int_{S_{s^{\prime} s^{\prime \prime}}} \frac{\gamma_{s^{\prime} s^{\prime \prime}}}{\alpha_{s^{\prime} s^{\prime \prime}}} A_{s s^{\prime}}(\boldsymbol{p}, t) A_{s s^{\prime \prime}}(\boldsymbol{q}, t) \mathrm{d} S \\
D(\boldsymbol{k}, t)=-\sum_{s^{\prime}, s^{\prime \prime}}\left[\int_{S_{s^{\prime} s^{\prime \prime}}} \frac{g_{s^{\prime} s^{\prime \prime}}}{\alpha_{s^{\prime} s^{\prime \prime}}} e(\boldsymbol{p}, t) \mathrm{d} S+\mathrm{i} \int_{\mathbb{R}^{3}} \frac{g_{s^{\prime} s^{\prime \prime}}}{F_{s^{\prime} s^{\prime \prime}}} e(\boldsymbol{p}, t) \mathrm{d}^{3} \boldsymbol{p}\right] .
\end{gathered}
$$

Going back to variables $e, Z, h$ from their definitions (2.18) one finds (omitting the obvious explicit time dependency of the spectra)

$$
\begin{aligned}
& T^{(e)}=\sum_{s^{\prime} s^{\prime \prime}} \int_{S_{s^{\prime} s^{\prime \prime}}} \frac{g_{s^{\prime} s^{\prime \prime}}}{\alpha_{s^{\prime} s^{\prime \prime}}}\left[e(\boldsymbol{p})(e(\boldsymbol{q})-e(\boldsymbol{k}))+s^{\prime} h(\boldsymbol{p})\left(s^{\prime \prime} h(\boldsymbol{q})-h(\boldsymbol{k})\right)\right] \mathrm{d} S \\
& T^{(h)}=\sum_{s^{\prime} s^{\prime \prime}} \int_{S_{s^{\prime} s^{\prime \prime}}} \frac{g_{s^{\prime} s^{\prime \prime}}}{\alpha_{s^{\prime} s^{\prime \prime}}}\left[s^{\prime} h(\boldsymbol{p})(e(\boldsymbol{q})-e(\boldsymbol{k}))+e(\boldsymbol{p})\left(s^{\prime \prime} h(\boldsymbol{q})-h(\boldsymbol{k})\right)\right] \mathrm{d} S
\end{aligned}
$$

and $T^{(Z)}=Z D(\boldsymbol{k}, t)$, using $\gamma_{s^{\prime} s^{\prime \prime}}(\boldsymbol{k}, \boldsymbol{p}, \boldsymbol{q})=\left[g_{s^{\prime} s^{\prime \prime}}(\boldsymbol{k}, \boldsymbol{p}, \boldsymbol{q})+g_{s^{\prime \prime} s^{\prime}}(\boldsymbol{k}, \boldsymbol{q}, \boldsymbol{p})\right] / 2$ in $(3.9)$. In addition to the gradient term $\alpha_{s^{\prime} s^{\prime \prime}}$, these transfer integrals only involve a single geometric factor $g_{s^{\prime} s^{\prime \prime}}$ explicited in equation (D 2).

\subsection{Preliminary comments about AQNM final equations}

The transfer terms $T^{(e)}$ and $T^{(h)}$ exactly set the rates of change $\partial e / \partial t$ and $\partial h / \partial t$ in the inviscid limit. They look similar to those derived by Galtier, 2003, but geometric coefficients may differ, according to the way integrands are symmetrized in terms of $\boldsymbol{p}$ and $\boldsymbol{q}$. Although a non zero viscous-damping coefficient $d=\mu_{k p q}$ has to be considered in order to correctly derive the asymptotic limit, this $d$ term no longer appears in the final equations.

Strong anisotropy results from the angular-dependent $\alpha_{s^{\prime} s^{\prime \prime}}$ term and from the topology of the resonant surfaces themselves; both effects reflect the appearance of a Dirac function $\delta\left(F_{s^{\prime} s^{\prime \prime}}\right)$ in energy and helicity transfers (see also Galtier, 2003). Because of the link of $\alpha_{s^{\prime} s^{\prime \prime}}$ to the group velocity, the transfers scale as $1 / \Omega$, which therefore replaces the eddy damping time scale $1 / \mu_{k p q}$ in classical EDQNM models with no rotation.

In the strict limit $\cos \theta=0$, the resonant surfaces approach the horizontal plane and a vertical plane, so that only planar triads are involved for $e$ and $h$, as in pure two- 
dimensional turbulence. This result is consistent with the first-order decoupling of the purely two-dimensional mode observed by Waleffe, 1993. Nevertheless, we have seen that the AQNM energy transfer always scales as $1 / \Omega$, whereas the rotation term disappears in the purely two-dimensional case. In addition, the geometric coefficient $g_{s^{\prime} s^{\prime \prime}} / \alpha_{s^{\prime} s^{\prime \prime}}$ in AQNM at $\cos \theta=0$ does not coincide with its counterpart in, e.g., two-dimensional EDQNM (Leith, 1971).

The transfer $T^{(Z)}$ in AQNM is linear in $Z$, and is the only term which does not reduce to a surface integral. Much more complex quadratic interaction terms that involve $Z$ in volumic $\mathrm{EDQNM}_{2-3}$ models are discarded in AQNM when removing rapidly oscillating terms. The latter approximations, used in subsection 3.1, are valid if $e^{\mathrm{i} \alpha \sigma_{k} t}$ with $\alpha=$ $s_{3}+s_{4}-s-s^{\prime}= \pm 2, \pm 4$ are really rapidly oscillating terms. They are questioned if $\cos \theta=k_{\|} / k$ is small enough, with a value of the same order as the inverse of the rotation frequency, since $\sigma_{k}=2 \Omega \cos \theta_{k}$. Accordingly, there exists a domain in which AQNM equations are no longer valid. As a consequence, the $A_{s s^{\prime}}$ have to be governed by different equations in the inner 'slow manifold' and in the outer domain; hence these 'slow amplitudes' can vary rapidly in the vicinity of $k_{\|}=0$, so that the slow/fast time separation is no longer valid and the resonance condition does not hold for the 'slow' manifold. This issue is further discussed in section 6.2 and in Cambon et al., 2004b.

\section{Numerical scheme for the energy equation}

From the previously introduced dynamical equations, we retain only the energy equation $e$, since we pick $h=Z=0$ initially, and the equations for $h$ and $Z$ imply that they remain zero. The following Lin type equation has to be solved

$$
\frac{\partial e}{\partial t}+2 \nu k^{2} e=\sum_{s^{\prime} s^{\prime \prime}} \int_{S_{s^{\prime} s^{\prime \prime}}} \frac{g_{s^{\prime} s^{\prime \prime}}(k, p, q)}{\alpha_{s^{\prime} s^{\prime \prime}}(\boldsymbol{p}, \boldsymbol{q})} e(\boldsymbol{p}, t)[e(\boldsymbol{q}, t)-e(\boldsymbol{k}, t)] \mathrm{d}^{2} \boldsymbol{p} .
$$


This equation is dimensional, and its dimensional analysis, with related definition of key non-dimensional timescales, can be obtained as follows. The wavenumber is nondimensionalized by $K_{0}$, which is an inverse lengthscale; the energy spectrum $e$ is scaled by an energy density amplitude $A$. A typical velocity scale is $u^{\prime} \sim A^{1 / 2} K_{0}^{3 / 2}$; instead of defining a typical time scale by $L / u^{\prime} \sim A^{-1 / 2} K_{0}^{-5 / 2}$, the scaling of the nonlinear timescale derives from the scaling of equation (4.1). The ratio $g_{s^{\prime} s^{\prime \prime}} / \alpha_{s^{\prime} s^{\prime \prime}}$ scales as $\pi K_{0}^{3} / \Omega$, so that the transfer term can be evaluated by $T^{(e)} \sim \pi K_{0}^{5} A^{2} / \Omega$, and from $e_{, t} \sim \frac{A}{\hat{t}_{N L}} \sim \pi \frac{K_{0}^{5}}{\Omega} A^{2}$ the typical nonlinear timescale is derived

$$
\hat{t}_{N L}=\frac{\Omega}{\pi A K_{0}^{5}} .
$$

On the other hand, a classical Rossby number is defined as $R o \sim u^{\prime} /(\Omega L)$ or

$$
R o=A^{1 / 2} K_{0}^{5 / 2} \Omega^{-1}
$$

so that $\Omega t \sim R o^{-2}$ for $t \sim \hat{t}_{N L}$. Thus, the evolution time for $e$ implied by (4.1) is $\mathcal{O}\left(R o^{-2} \Omega^{-1}\right)$.

One can distinguish three domains depending on the elapsed time. At shortest times, $\Omega t \sim 1$, nonlinearity is negligible, and $e$ is conserved. At larger times, $\Omega t \sim R o^{-1}$, one recovers the timescale which is relevant for classical nonlinear dynamics, e.g. related to conventional energy transfer in terms of volume integral. Only at the largest times, $\Omega t \sim R o^{-2}$, can the 'weak' cascade establish in inertial wave-turbulence, and $e$ evolve.

The previous analysis is essentially inviscid. If the viscous term is accounted for in equation (4.1), only for numerical convenience, a fictitious Reynolds number can be defined as $R e=\pi A k_{0}^{3} /(2 \Omega \nu)$. Since the typical time scaling $t / \hat{t}_{N L}$ is used, the Rossby number has disappeared at this point, and an apparently low value of the Reynolds number is found.

Starting from a pure three-dimensional isotropic case, with $e=E(k) / 4 \pi k^{2}$, an angular 
dependent $e(k, \theta, t)$ distribution is created by the anisotropic energy transfer. The viscous term is marginal in our study since we are interested in the limit of infinite Reynolds number and vanishing Rossby number. Non-zero viscosity will be introduced only for numerical convenience. Since we have used an Euler scheme for time marching, the viscous term in (4.1) can be readily added in an implicit way by using integrating factors.

\section{Numerical implementation, parameters}

First of all, from equation (4.1) we note that if the energy spectrum is initially axisymmetric or contains mirror symmetry, it conserves this property. This is used for reducing the number of degrees of freedom in the computation. Singularities due to the cancelling of the denominator $\alpha_{s^{\prime}} s^{\prime \prime}$ are removed by using symmetries of the integrand (see Bellet, 2003 for details).

The integral in (4.1) can be recast in terms of the normalized wavevectors $\boldsymbol{K}=\boldsymbol{k} / k$, $\boldsymbol{P}=\boldsymbol{p} / k$ and $\boldsymbol{Q}=\boldsymbol{q} / k$. Without loss of generality $\boldsymbol{K}$ 's components are $K_{1}=\sin \theta$, $K_{2}=0, K_{3}=\cos \theta$. The second leg of the triad is parameterized in spherical coordinates with an exponential radius as $\boldsymbol{P}=\exp (\rho)\left[\sin \theta^{\prime} \cos \phi^{\prime}, \sin \theta^{\prime} \sin \phi^{\prime}, \cos \theta^{\prime}\right]$, and $\boldsymbol{Q}$ comes as the difference $\boldsymbol{K}-\boldsymbol{Q}$. The choice of an exponential radial parameter simplifies the computation of the geometric coefficients. In view of the symmetries of the integral, the triplet is such that $\rho \in]-\infty, \infty\left[, \theta^{\prime} \in\right] 0, \pi\left[\right.$ and $\left.\phi^{\prime} \in\right] 0, \pi[$.

This parameter space for $\boldsymbol{P}$ is discretized using $M_{\rho}$ values for $\rho$-which yield $P \in$ $\left[k_{m}, k_{M}\right]-, M_{\theta}$ polar angles and $M_{\phi}$ azimuthal ones. The intersection of each elementary volume of this grid with the surface $S_{s^{\prime} s^{\prime \prime}}$ is obtained by inspection of the sign of the characteristic function $f_{s^{\prime} s^{\prime \prime}}\left(\rho, \theta^{\prime}, \phi^{\prime}\right)=\cos \theta+s^{\prime} P_{3} / P+s^{\prime \prime} Q_{3} / Q$ over each vertex of the elementary volume cell. A change of sign indicates that the resonant surface intersects the cell, and the contributed area is computed by a first-order estimate, assimilating locally the surface to a plane. This allows one to compute the geometrical coefficients 
in the energy integral of (4.1). Knowing the numerical values of the energy $e$ at the preceding time step over the grid points, one still has to obtain the energy for $\boldsymbol{Q}$ which do not necessarily lie on the grid. The needed $e(\boldsymbol{Q})$ is interpolated with a two-dimensional second-order scheme using the values at neighbouring points.

Choosing figures for $M_{\rho}, M_{\theta}$ and $M_{\phi}$ is not easy, and we start with the value 100 for each and let them vary to check the effects of discretization (see appendix E for details). We then study the dependence of the numerically integrated energy transfer, which should cancel out over the whole spectral space due to energy conservation:

$$
\int_{0}^{\infty} k^{2}\left(\int_{0}^{\pi} T(k, \theta) \sin \theta \mathrm{d} \theta\right) \mathrm{d} k=0 .
$$

Of course, the above integral is not exactly zero for a finite discretization and truncated $k$, but should be small, and converge to zero with increasing degrees of freedom. The dependence with $M_{\rho}$ is the strongest, which we estimate to be with a power between one and two, as illustrated by figure 2 .

A three-dimensional plot of a resonant surface for $\theta=1.4$ is provided on figure 3 , showing the reader how complex resonant surfaces can get, exhibiting cusps at the connection of the different folds. When $\theta$ varies between 0 and $\pi / 2$, the surface shape changes, the closed loops eventually becoming infinite.

\section{Numerical results}

Solving numerically the AQNM energy equation with accuracy and trust has been the main challenge of this study. To our knowledge, this was never done in the WaveTurbulence community. Given the complexity of resonant surfaces, numerical integration has to involve a huge number of degrees of freedom; related matrices have a typical size, for instance $300^{3}$ at the maximum, which can be of the same order of magnitude as for pseudo-spectral DNS. The important computational resources called into play to solve 
the AQNM energy equation were also used to perform new runs of the volumic EDQNM23 code, with much better conditions (spatial resolution, number of time-steps) than in any previous computation.

In contrast with the numerical cost of a single typical AQNM run, the parametric study is dramatically reduced. Since AQNM addresses the asymptotic limit of vanishing Rossby number, there is no need for different values of the Rossby number. The limit of an infinite Reynolds number is mainly addressed too, and viscosity is reintroduced only for numerical convenience, yielding very few different runs for different Reynolds numbers.

\subsection{Time evolution of the energy and related transfer}

AQNM equation (4.1) is solved starting from an isotropic narrow-band energy spectrum, as usual in DNS and EDQNM (Orszag, 1969). During a first phase, without viscosity, an inertial zone develops, and extends towards larger and larger wavenumbers. At a time $t_{f}\left(k_{\max }\right)$, the inertial zone reaches the largest wavenumber of the mesh, denoted $k_{\max }$, and the tail of the energy spectrum begin to display a bump, which can eventually create a numerical instability. A laminar viscous term is added to remove this bump, and to allow us to continue the calculation at larger times. In this sense, viscosity is a numerical artifact, as well as the low value of the typical Reynolds numbers defined below is not physically relevant.

\subsubsection{Distribution of the spectral density of energy and related transfer}

We first consider the energy spectrum $E(k, t)$ which derives from $e(k, \cos \theta, t)$ by spherical integration over $\theta$ of the energy density spectrum:

$$
E(k, t)=4 \pi k^{2} \int_{0}^{1} e(k, x, t) \mathrm{d} x, \quad x=\cos \theta .
$$


Figure 5 shows the evolution of $E(k)$ starting from the narrow band spectrum we have picked for as the initial condition. One identifies two stages in the time evolution of the spectrum. First, as shown on figure 5a, the spectrum widens until it reaches the smallest scales consistent with the dynamics at the given Reynolds number $R e=5$. The corresponding time is approximately $t=0.525$. At this point, the dissipative subrange is completely operative, and the spectrum begins to decay so that the system globally loses energy, as shown in figure 5b. As the first remarkable result, the inertial range establishes naturally with a clear $k^{-3}$ power law for $E(k)$. This scaling of the inertial range was identified in rotating turbulence in forced numerical simulations by e.g. Smith \& Waleffe, 1999, Hossain, 1994, recovered in high resolution freely decaying $\mathrm{EDQNM}_{2,3}$ with the highest resolution (Bellet, 2003), and in LES by Yang \& Domaradzki, 2004.

The build-up of the angular dependence is illustrated by iso-values of $e$ in the $(k, \theta)$ plane, plotted in figure 7. The initial distribution (shown on figure 7a) is isotropic, so that the contours are straight lines parallel to the $\theta$ axis, showing no dependency of the spectrum with the angle $\theta$. Almost instantaneously after $t=0$ (figure $7 \mathrm{~b}$ ), the spectrum begins to spread in the angular direction, showing an exchange of energy between different inertial waves of different propagation angle, as an adjustment of the dynamics of turbulence under the effect of the Coriolis force. Of course, energy is also transfered between different wavenumbers, as observed in figures $7 \mathrm{c}$ to $7 \mathrm{e}$, at which point the angular equilibrium of the spectrum is almost reached. Finally, the last figure $7 \mathrm{f}$ shows that $e$ is large in the neighbourhood of the horizontal plane $\theta=\pi / 2$, whereas only a weak angular dependence of the energy density spectrum is observed away from this region. This distribution is qualitatively very similar to the one found in the work by Cambon et al., 1997 (their figure 6), both from $\mathrm{EDQNM}_{2}$ results and large eddy simulation of homogeneous turbulence with rotation. 
An additional viewpoint on the energy density distribution is obtained by plotting the compensated spectrum $4 \pi k^{2} e(k, \cos \theta, t)$ versus $k$, at different fixed angles $\theta$ as done on figures $8 \mathrm{a}$ and b. All curves collapse on $E(k)$ for isotropic turbulence (e.g. here at $t=0$ ). For angles close to $\pi / 2$, the slopes are significantly less steep than $k^{-3}$, about $k^{-2}$, whereas they are steeper than $k^{-3}$ for angles far from $\pi / 2$. It is therefore clear that the $k^{-3}$ law for $E(k, t)$ results from averaging over all angles. Figures $8 \mathrm{c}$ and $\mathrm{d}$ represent the same quantity multiplied by $\sin \theta$, which is the weighting factor which appears in the integral when computing $E(k, t)$. Figures $8 \mathrm{a}$ and $\mathrm{c}$ show the distribution at $t=0.525$ and $8 \mathrm{~b}$ and $\mathrm{d}$ at $t=1.05$, from which we notice that the level of the close to horizontal spectrum has increased in between times.

Comparing either figures $8 \mathrm{a}$ and $\mathrm{b}$ or $8 \mathrm{c}$ and $\mathrm{d}$ indicates a striking similarity between the spectra at different times. More detailed study shows that, leaving aside the high wavenumber components which are affected by viscosity and the highest of the curves (very nearly horizontal wavectors), spectral evolution is self-similar. This is best observed in figure $8 \mathrm{e}$ which combine plots $8 \mathrm{c}$ and $\mathrm{d}$ on a zoomed scale. Whereas the maximum energy density for the angle close to the horizontal hardly changes, the remaining curves fall together with increasing time. It therefore appears that the large-time behaviour is self-similar outside a neighbourhood of $\theta=\pi / 2$ whose width decreases to zero. Figure 9 confirms this conclusion.

In isotropic turbulence, the direction over which spectra are integrated in spectral space to yield one-dimensional spectra does not modify the $k^{-5 / 3}$ power law of the inertial range. However, the same integration of the anisotropic spectrum $e(k, \cos \theta)$ over horizontal planes in spectral space, or over vertical cylinders modifies the scaling of the resulting one-dimensional spectrum, as exhibited on the plot 10(a) and (b) respectively. In the one-dimensional horizontal spectra $E_{h}\left(k_{\|}\right)$and $E_{h}\left(k_{\perp}\right)$, the scaling is not $k^{-3}$ 
whereas this scaling describes the vertical spectra $E_{v}\left(k_{\|}\right)$and $E_{v}\left(k_{\perp}\right)$. This is due to the specific distribution in spectral space directions of the energy density $\operatorname{spectrum} e(k, \cos \theta)$.

A way of quantifying the angular distribution of energy is to compute the energy at each angle integrated over every wavenumber

$$
E_{\text {ang }}(\theta, t)=2 \pi \int_{0}^{\infty} k^{2} e(k, \theta, t) \mathrm{d} k
$$

such that the total energy is recovered as $E_{t}(t)=\int_{0}^{\pi} E_{\text {ang }}(\theta, t) \sin \theta \mathrm{d} \theta$. Another interesting quantity is the fraction of the total energy contributed by angles between $\theta$ and $\pi-\theta:$

$$
E_{\mathrm{frac}}(\theta, t)=E_{t}^{-1} \int_{\theta}^{\pi-\theta} E_{\mathrm{ang}}(\theta, t) \sin \theta \mathrm{d} \theta
$$

The two quantities $E_{\text {ang }}$ and $E_{\text {frac }}$ are plotted in figure 11 . Their time evolution is shown, starting with isotropic initial conditions, for which $E_{\text {ang }}(\theta)=E_{t} / 2$ and $E_{\text {frac }}=$ $\cos \theta$. Figure 11 a shows that $E_{\text {ang }}$ grows rapidly for $\theta$ close to $\pi / 2$, developing a sharp peak for horizontal wavevectors and a distribution which is far from isotropic at the end of the computation. Figure $11 \mathrm{~b}$ also shows the development of anisotropy and that $E_{\text {frac }}(\theta, t)$ appears to approach a limit $E_{\text {frac }}^{\infty}(\theta)$ at large times, reflecting the self-similarity discussed earlier. According to these results, $50 \%$ of the energy at the end of the run is contained between $\theta=0$ and $\theta=1.25$. Taken together, figures 11a and $11 \mathrm{~b}$ indicate that, although $E_{\text {ang }}$ appears to develop an infinite singularity at $\theta=\pi / 2$, this singularity is integrable and hence does not dominate the overall energy.

In the same way these angular dependent quantities have been defined for the energy, one may compute a similar function from the energy transfer spectrum, by merely replacing $e(\theta, k, t)$ by $T(\theta, k, t)$ in equation (5.1), thus yielding $T_{\text {ang }}(\theta, t)$. From this quantity, one obtains the energy flux across a cut at $\theta: E_{\text {flux }}(\theta, t)=\int_{\theta}^{\pi / 2} T_{\text {ang }}(\theta, t) \sin \theta \mathrm{d} \theta$. 
Figure 13 shows how this quantity evolves in time: the energy flux is maximal at the beginning of the computation, but its maximum is shifted when time increases towards the horizontal plane $\theta=\pi / 2$, reaching almost 1.5 .

\subsection{Time evolution of total kinetic energy and of single-point indicators of anisotropy}

The modification of the spectral distribution of energy density in rotating turbulence is a marker of the structuration of the flow, and of its modified dynamics with respect to isotropic turbulence. Upon integration of the energy density spectrum over the complete spectral space, one obtains the total energy $E_{t}=q^{2} / 2$ in the flow, whose decay is plotted on figure $6 \mathrm{a}$. The power obtained at the end of our computation is -0.8 , which shows a strong reduction of energy transfer and hence of the decay rate of turbulence with respect to the isotropic law $t^{-10 / 7}$. This was again observed by Cambon et al., 1997and Squires et al., 1994. Comparing with the result obtained from the $\mathrm{EDQNM}_{2}$ model run in the same conditions, we observe on figure $6 \mathrm{~b}$ that the decay rate of both models is very similar, since the $\mathrm{EDQNM}_{2}$ model yields a $t^{-0.86}$ power law.

The large-time, inviscid self-similarity identified earlier can be expressed more precisely by

$$
\frac{e\left(k_{s}(t) \kappa, \theta, t\right)}{e_{s}(t)} \rightarrow f(\kappa, \theta)
$$

at infinite Reynolds number as $t \rightarrow \infty$, for any fixed values of the scaled wavenumber $\kappa=k / k_{s}$ and $\theta \neq \pi / 2$, where $k_{s}(t), e_{s}(t)$ are appropriate scales for wavenumber and energy density. If such limiting behaviour is assumed for $e$ and the resulting expression,

$$
e(\boldsymbol{k}, t)=e_{s}(t) f\left(\boldsymbol{k} / k_{s}(t)\right)
$$

is used in the AQNM equation (4.1) (without the viscous term), the wavenumber and energy-density scales can be shown to evolve according to the power laws

$$
e_{s}(t) \propto\left(t+t_{0}\right)^{5 \alpha-1},
$$




$$
k_{s}(t) \propto\left(t+t_{0}\right)^{-\alpha}
$$

As a result, the total energy, given by the spectral-space volume integral of $e$, behaves like $\left(t+t_{0}\right)^{2 \alpha-1}$. Figure 6 , discussed above, shows that there is indeed a power law, a result which supports the conclusion of self-similarity and whose exponent of -0.8 for the total energy implies $\alpha=0.1$. Note that such a link between inviscid self-similarity and temporal power laws is a classical one for nonrotating, isotropic turbulence (see e.g. Monin \& Yaglom, 1975, section 16.1) and that, without self-similarity, a power law is hard to explain. Note also that the scaled, limiting spectrum $f(\kappa, \theta)$ appears to have an integrable singularity at $\theta=\pi / 2$.

The full Reynolds stress tensor involves both a spherical and a deviatoric part as $<u_{i} u_{j}>=q^{2}\left(\delta_{i j} / 3+b_{i j}\right)$, the latter deviatoric tensor $b_{i j}$ reflecting anisotropy. Because of the symmetry — axisymmetry without mirror symmetry — , the deviatoric tensor can be expressed from only one component, say $b_{33}$, as $b_{i j}=\left(-3 b_{33} / 2\right)\left(\delta_{i j} / 3-\delta_{i 3} \delta_{j 3}\right)$. In agreement with the relationship (B 3) in Appendix B, $b_{i j}$ is in general the sum of two different contributions $b_{i j}^{e}$ and $b_{i j}^{z}$, which reflect the directional and the polarisation anisotropy respectively. This decomposition here reduces to

$$
q^{2} b_{33}=\int\left[\left(e-\frac{E}{4 \pi k^{2}}\right) \sin ^{2} \theta+\Re\left(Z e^{-2 \mathrm{i} \sigma t}\right) \sin ^{2} \theta\right] d^{3} \boldsymbol{k}
$$

with $b_{i j}=b_{i j}^{e}$ in the absence of $Z$-contribution. As shown in figure $14, b_{33}^{e}$ starts from zero (3D isotropy) and saturates at a value close to $0.07-0.08$. This value is about half the one expected for a pure 2D flow, or $b_{33}^{e}=1 / 6$, and recovered in (5.7) using for $e$ a Dirac function $e=\frac{E(k, t)}{2 \pi k} \delta\left(k_{\|}\right)$(details in CMG, section 4). Such a saturated value is also obtained in recent DNS/LES of rotating flows (Morinishi et al., 2001a, Morinishi et al., 2001b, Yang \& Domaradzki, 2004) in which $b_{i j}^{e}$ is plotted, following CMG (their figure 7). in some DNS/LES results, however, this growth of $b_{33}^{e}$ is counterbalanced by 
a stronger decrease of $b_{33}^{z}$, resulting in a negative value of $b_{33}$. This will be discussed in the next section.

\section{General discussion}

Turbulence in a rotating frame illustrates the subtle interplay between linear and nonlinear processes and the significance of spectral anisotropy, especially the angular dependence of spectral energy which reflects dimensionality.

The breaking of isotropy is potentially present in the linear operator since the dispersion law of inertial waves depends on their angle of propagation, not to mention the anisotropic definition of the helical modes. Nevertheless, this potential anisotropy is not necessarily reflected on statistical quantities. The second order spectral tensor-singletime two-point velocity correlations-remains isotropic in the linear regime, as shown by the system of equations (2.19)-(2.21) with zero right-hand-side. A possible breaking of isotropy through two-time velocity correlations is not discussed here for the sake of consistency (see Cambon et al., 2004a). Going back to equation (2.19), it is clear that isotropy is broken in the energy equation by the energy transfer term through single-time triple correlations. Isotropy breaking in our case firstly comes from the linear operators which act on triple velocity correlations as a product of three Green's functions (e.g. equation 2.25).

\subsection{Directional anisotropy}

In addition to the detailed dependence of the energy spectra with the wavenumber $k$ and the angle $\theta$, the AQNM model yields a $k^{-3}$ scaling for the inertial spectral subrange. This law is very different from the one found in $2 \mathrm{D}$ turbulence, which corresponds to $E^{2 D}\left(k_{\perp}\right) \sim \overline{\omega^{2}} k_{\perp}^{-3}$ with enstrophy $\left(\overline{\omega^{2}}\right)$ conservation. Our $E(k)$ is three-dimensional and

would reduce to the former only if $e=\frac{E(k)}{2 \pi k} \delta\left(k_{\|}\right)$. Even if some authors advocated two- 
dimensionalisation of turbulence by rapid rotation from forced under-resolved DNS (e.g. Hossain), and tried to interpret a $k^{-3}$ law along this line, our results are not consistent with two-dimensionalisation. Looking at the explicit $\theta$-dependent spectra, we propose that, since the horizontal wavevectors exhibit a decay exponent larger than -3 with $k$ whereas oblique ones decay with a smaller exponent, a combination thereof is necessary to achieve the $k^{-3}$ behaviour.

We note that the full $(k, \theta)$ distribution of the energy density spectrum gotten with AQNM is very much like that obtained in previous works with the EDQNM model and Large Eddy Simulations of rotating homogeneous turbulence. The spectral anisotropy is reflected in a specific anisotropic structuration of the flow when using specifically weighted integrals of the energy spectrum.

The reader is refered to Galtier, 2003 and CRG for a discussion of analytical laws obtained from WT with additional assumptions $\left(k_{\|} \ll k_{\perp}\right)$ compared with our AQNM numerical results. Galtier's proposal $e \sim k_{\|}^{-1 / 2} k_{\perp}^{-7 / 2}$ is consistent with the $k^{-2}$ law for $k^{2} e(k, \theta, t)$ at the smallest $\cos \theta=k_{\|} / k$ (figure 8 ), but a significant inverse cascade is found by AQNM in this domain, which is not allowed by Galtier's analysis. Also on figure 11(a), the curve representing $(\cos \theta)^{-1 / 2}$ suggested by Galtier's analysis is plotted against the AQNM results.

\subsection{Relevance of $A Q N M$ and the role of the quasi-slow manifold}

As discussed earlier, the asymptotics leading to (4.1) suffer from non-uniformity for $\boldsymbol{k}$ near the horizontal plane. This comes about because (2.22) with (3.1) consists of oscillatory exponential terms of frequency $\left(s_{3}+s_{4}-s-s^{\prime}\right) \sigma_{k}$. For $\boldsymbol{k}$ which are not close to the horizontal plane, these exponentials are rapidly oscillatory, and hence dropped in the asymptotic analysis, unless $s_{3}+s_{4}=s+s^{\prime}$. However, if $\boldsymbol{k}$ is close to horizontal, $\sigma_{k}$ is small and the given terms are no longer rapidly oscillating. As a result, there is a small 
region of angles near $\theta=\pi / 2$ in which a more complete asymptotic description should be used.

Despite the above non-uniformity, the model provides a self-consistent description of wavevectors away from the horizontal plane, because it predicts only an integrable singularity in the energy density. Whether or not the near-horizontal region is important depends on which statistical quantity is required. For instance, the spherically averaged spectrum and total energy will be negligibly affected, whereas other quantities depend on the details of near-horizontal wavenumber spectra. For example, the integral lengthscales with vertical separation defined by (Jacquin et al., 1990)

$$
\begin{gathered}
\overline{u_{1}^{2}} L_{11}^{(3)}=\overline{u_{2}^{2}} L_{22}^{(3)}=\pi^{2} \int_{0}^{\infty}(e(k, \cos \theta=0)-Z(k, \cos \theta=0)) k d k \\
\overline{u_{3}^{2}} L_{33}^{(3)}=2 \pi^{2} \int_{0}^{\infty}(e(k, \cos \theta=0)+Z(k, \cos \theta=0)) k d k .
\end{gathered}
$$

AQNM is capable of predicting any statistical quantity of the first kind; the current results for the spherically averaged energy spectrum and in section 5.2 for the Reynolds stress tensor are unlikely to be modified by the refined analysis of the slow manifold. An important feature of the extended WT theory is the emergence of the polarisation anisotropy $Z$, which is therefore zero almost everywhere but in the slow manifold, as well as in our case where AQNM is started with 3D isotropy. It can therefore be inferred that the contribution of $Z$ is unimportant for deriving e.g. the Reynolds stress tensor, but is essential if the behaviour of typical integral lengthscales is concerned.

A possible means of treating the near-horizontal wavevectors is discussed in CRG with a proposal for using matched expansions with a specific inner model for the slow manifold, and AQNM as the outer model. The way to perform this analysis is hinted by the general $\mathrm{EDQNM}_{2-3}$ model (detailed $\mathrm{EDQNM}_{3}$ equations in CRG appendix) which underlies the AQNM model. 
A nonlinear Proudman theorem is often invoked, which means the independence of the slow manifold, which could be governed by its own equations in accordance with autonomous 2D dynamics. This argument is supported by mathematical developments for some classes of initial data (Babin et al., 1999), and not by others (Babin et al., 2001). It is important to point out that periodicity conditions are used, and that the possible decoupling of the $2 \mathrm{D}$ manifold from the rest is only valid at the first order. Our AQNM approach is essentially different since our equations (and those of Galtier, 2003) were found in a continuous unbounded domain, excluding the exact $2 \mathrm{D}$ manifold. This 'nonlinear Proudman theorem' is either irrelevant, if the slow mode is unimportant because of its integrable singularity, or questioned at largest times, if the slow mode is analysed by itself and/or for specific quantities which rely only upon it. In this second case, the analysis introduced by CRG, even if not analytically developed, demonstrate a typical coupling between the slow and rapid modes through new volume and principal value integrals, with respect to AQNM. These coupling terms are neglected in AQNM when discarding those terms considered as rapidly oscillating.

\section{3. $2 D$ or not $2 D$ ?}

As we have seen, our results indicate the transfer of energy towards horizontal wavevectors and also the appearance of large energy densities near the horizontal plane. At first glance, this suggests the possibility that the flow becomes two-dimensional. However, this is not the case for the following reasons. Firstly, following the development phase and hence at large times, the spectrum evolves in a self-similar manner (recall figures 8 and 9). This outcome excludes two-dimensionalization in the limit of large time. Secondly, as indicated by figure 11, the overall energy is not dominated by horizontal wavevectors as it would be for a two-dimensional flow. Figure 11 also illustrates the singularity in the energy density. 
DNS and LES results have also shown the tendency of rotating turbulence to become anisotropic by transfer towards the horizontal plane. For instance, Bartello et al., 1994 showed the development of vortices elongated in the vertical direction, while Cambon et al., 1997 determined the time evolution of spectra leading to results comparable with those of figure 7. However, it is difficult to decide, based on these results, whether the flow does or does not become two-dimensional in the large-time limit and this for several reasons. Spatial periodicity of the flow, which is assumed numerically, implies that the size of the periodic box must be sufficiently large in order to avoid effects of numerical confinement. In particular, the turbulent correlation length should be small compared with the box size, as should $c_{g} t$ where $c_{g}$ is the group velocity associated with inertial waves. The latter condition becomes harder to satisfy the longer the time simulated. Since the evolution time at small Rossby number scales as $R o^{-2} \Omega^{-1}$, the limit of small Ro is particularly hard to treat.

\subsubsection{Reynolds stress tensor anisotropy}

As concerns Reynolds stress tensor (RST) anisotropy with directional/polarisation splitting, recent DNS/LES (CMG, Morinishi et al., 2001a, Yang \& Domaradzki, 2004) with correct resolution show results similar to those of AQNM for the $b_{33}^{e}$ history, also in agreement with $\mathrm{EDQNM}_{2}(\mathrm{CMG})$. The maximum value eventually reached is never larger than 0.08 , i.e. very close to the AQNM limit, and therefore remained far from the theoretical 2D limit which is $1 / 6$. In addition, a rapid evolution of $b_{33}^{z}$ can yield a strong departure of $b_{33}$ from $b_{33}^{e}$, resulting eventually on a negative value of $b_{33}$ (Yang \& Domaradzki, 2004). Even if a significant value of $Z$ is expected in the slow manifold (see CMG and CRG), in agreement with the behaviour of the integral lengthscales with vertical separation, we find highly questionable that this term could contribute dominantly 
to the RST anisotropy. Hence, the significant negative value of $b_{33}^{z}$ which contaminates $b_{33}$ is probably due to the following causes:

(a) isotropy is not precisely controlled in the initial data, and strong departures from axisymmetry appear $\left(b_{11} \neq b_{22}\right)$ only after small elapsed times (Yang \& Domaradzki, 2004);

(b) the numerical box is too small given the huge elapsed time used, so that an artefactual confinement biases the most elongated scales;

(c) the angular-phase mixing in (5.7), due to the term $e^{-2 \mathrm{i} \sigma t}$, which can damp any $Z$ contribution is not accurately computed in DNS.

The previously mentioned drawbacks $a, b$ were not present in the LES database in CMG, with an aspect ratio of 4 (resolution $512 \times 128 \times 128)$ optimised to control the anisotropy (initial isotropy becoming axisymmetry) and to avoid artefactual confinement. Nevertheless, a second transition occurred, with $b_{33}^{z}$ becoming significantly negative, although $b_{33}$ itself never became negative. This result has to be considered with care, since the typical Rossby number based on the cut-off wavenumber became of the order of unity and unexpected oscillations appeared after the second transition. Note that in their $64^{3}$ DNS/LES, Bartello et al., 1994 found strong negative values of $b_{33}$, without using the $b_{i j}^{e}, b_{33}^{z}$ splitting. As a final comment, it is important to point out that a positive value of $b_{33}$ in true axisymmetrical turbulence means $a=<u_{\|}^{2}>/<u_{\perp}^{2}>$ larger than one $\left(a=2\right.$ if $\left.b_{33}=1 / 6\right)$ whereas a negative value of $b_{33}$ means $a$ smaller than one $(a=0$ if $\left.b_{33}=-1 / 3\right)$. This illustrates that dimensionality and polarization may have opposite effects on the RST anisotropy, or componentality (see also Reynolds \& Kassinos, 1995). In this prospect, DNS/LES may exhibit a two-component limit instead of a twodimensional one, due to low resolution, but also because the ensemble averages needed to obtain reliable statistics are not available from one realisation only. 
6.3.2. $k^{-3}$ slope

From the sole fact that the energy transfer scales as $1 / \Omega$, a $k^{-2}$ slope could be inferred for the spherically averaged energy spectrum (Zhou, 1995), but this oversimplified argument ignores anisotropy. In contrast, our results indicate $k^{-3}$ for the spherically averaged spectrum in the inertial range. According to the power laws, (5.5) and (5.6), arising from self-similarity of the 3D spectrum, the spherically averaged spectrum should behave as $t^{3 \alpha-1}$ at large time. Given $k^{-3}$ behaviour, this leads to an inertial-range spectrum like $t^{-1} k^{-3}$, with a purely numerical prefactor. This result, when expressed in dimensional terms, implies that the inertial-range, spherically averaged spectrum scales as $\Omega t^{-1} k^{-3}$, independent of the initial spectral parameters. Comparison of the spectra in figure 5b at $k=2$ and the two times $t=0.525$ and $t=1.05$ shows a decrease by a factor of very nearly 2 (to within a few percent). Such close correspondence with the $t^{-1}$ law provides further confirmation of the hypothesis of self-similarity.

Reviewing DNS/LES of decaying turbulence, it seems that only Yang \& Domaradzki, 2004 found a clear transition from $k^{-2}$ to $k^{-3}$, the latter result being in qualitative agreement with our AQNM results. Forced DNS/LES often suffer from numerical biases even worse than those in the free decay, so that only the study by Smith \& Waleffe, 1999 is discussed here, in which the forcing is truly isotropic and limited to a small scale region with wavenumber $k_{f}$. Before comparing the former results with our AQNM (numerical) ones, it is important to stress some different definitions of spectra. In order to avoid ambiguity, the spherically averaged spectrum and its counterpart near the 'slow manifold' will be referred to as $E^{N}(k)$ and $E^{N}\left(k_{\|}, k_{\perp}\right)$ in SW. It now appears that $E^{N}(k)$ is the same as our $E(k)$, but a prefactor $k_{\perp}$ has to be introduced to compare the second spectrum with our $e$, so that $e(k, \cos \theta) \sim k_{\perp} E^{N}\left(k_{\|}, k_{\perp}\right)$. Accordingly, the result of SW for the slow manifold, for $k$ smaller than $k_{f}$, or $E^{N}\left(k_{\perp}, k_{\|}\right) \sim k_{\perp}^{-3}$, is 
consistent with the law $k^{2} e(k, \cos \theta \sim 0) \sim k^{-2}$ shown in figure 8 for the smallest values

of $\cos \theta=k_{\|} / k=k_{\|} / \sqrt{k_{\|}^{2}+k_{\perp}^{2}}$, since $k \sim k_{\perp}$ in the spectral domain considered. On the other hand, the collapse of $E^{N}(k)$ and $E^{N}\left(k_{\|}=0, k_{\perp}\right)$ observed in SW at smallest $k$, interpreted as a sign of two-dimensionalisation of the largest scales, is not obtained in our AQNM results, so that the similar $k^{-3}$ laws obtained for both $E^{N}(\mathrm{SW})$ and $E$ (AQNM) likely have different causes. The results of SW have two consequences: an energy transfer from small to large scales was evidenced, although different from the classical pure 2D dynamics one; strong coupling between rapid and slow modes is shown, with no independence of the slow manifold. The scaling $E^{N}\left(k_{\|}=0, k_{\perp}\right) \sim k_{\perp}^{3}$ is confirmed by Smith \& Lee, 2005, restricting the calculation to near-resonant triads.

\section{Conclusion}

In this work, we propose a nonlinear model for the dynamics of inertial waves in the limit of high Reynolds number and low Rossby numbers. We show that the asymptotic behaviour of turbulence can be described independently of the explicit value of the rotation rate, viscosity only remaining as a means of regularizing the equations at the large wavenumber numerical cut-off. The derivation of the Asymptotic Quasi-Normal Model is started from the existing anisotropic Eddy-Damped Quasi-Normal statistical model, which includes parts of the Green's function influence onto second- and third-order velocity correlations, coming from inertial waves. Instead of integrating the energy transfer over all possible interaction triads of inertial waves, the AQNM model is simplified with respect to the EDQNM one by reducing the integration over resonant triads of inertial waves, which are shown to emerge in the limit of fast rotation. Thus, asymptotic analysis leads to a Lin type energy equation, with energy transfer computed over surfaces of resonant interactions only. Handling the complex topology of these surfaces is the price 
to pay for the simplification of the integral. The most important and original part of this work is that we propose a numerical implementation of the AQNM model, and get quantitative results for the evolution of the energy density spectrum, to be contrasted with the qualitative results obtained in previous wave turbulence studies through assumptions of a specific scaling of the spectra.

The model predicts a development phase in which both anisotropy and an inertial range appear. Subsequently, spectral power laws are found in which the spherically averaged inertial range spectrum behaves like $k^{-3}$. However, this represents the average of power laws whose exponent depends on the wavevector direction: the exponent runs form -2 to -4 depending on the angle considered. The spectral evolution is observed to be self-similar at large times, an outcome which excludes two-dimensionalization of the flow in the limit $t \rightarrow \infty$. The energy density is found to be large for nearly-horizontal wavevectors, apparently reflecting a singularity at the horizontal plane. However, this singularity is integrable, and so quantities like the spherically averaged spectrum and Reynolds stresses are not dominated by this singularity. This may be contrasted with the two-dimensional case for which the horizontal plane contains all the energy. Finally, the kinetic energy decays as $t^{-0.8}$.

The numerical part of the work was possible thanks to parallel computing time offered by the following national computing centers: Centre Informatique National de l'Enseignement Supérieur (CINES, Montpellier); Commissariat à l'Énergie Atomique (CEA-CENG, Grenoble); Institut du Développement et des Ressources en Informatique Scientifique (IDRIS, Orsay). 


\section{Appendix A. Poloidal-toroidal decomposition, and Craya-Herring frame of reference}

The poloidal-toroidal decomposition is used to represent a three-component divergencefree velocity field in terms of two independent scalar terms, taking advantage of the presence of a privileged direction $\boldsymbol{n}$.

$$
\boldsymbol{u}=\boldsymbol{\nabla} \times\left(s_{\text {pol }} \boldsymbol{n}\right)+\boldsymbol{\nabla} \times\left[\boldsymbol{\nabla} s_{\text {tor }} \times \boldsymbol{n}\right]
$$

the axial vector $\boldsymbol{n}$ being chosen along the vertical direction, without loss of generality. As a caveat, some care is needed to represent a Vertically Sheared Horizontal Flow (VSHF hereinafter, after Smith \& Waleffe, 2002) or $\boldsymbol{u}(\boldsymbol{x} \cdot \boldsymbol{n}, t)$, with $\boldsymbol{u} \cdot \boldsymbol{n}=0$, with this decomposition.

In Fourier space, the above decomposition yields a pure geometrical one, or

$$
\hat{u}=\boldsymbol{k} \times \boldsymbol{n}\left(\hat{\mathrm{i}}_{\text {pol }}\right)-\boldsymbol{k} \times(\boldsymbol{k} \times \boldsymbol{n})\left(\hat{s}_{\text {tor }}\right)
$$

and it appears immediately that the Fourier mode related to vertical wavevector direction, or $\boldsymbol{k} \| \boldsymbol{n}$, has zero contribution; this 'hole' in the spectral description yields missing the VSHF mode in physical space. In order to complete the decomposition, one can define an orthonormal frame of reference, which is nothing else than the local reference frame of a polar-spherical system of coordinates for $\boldsymbol{k}$ :

$$
\boldsymbol{e}^{(1)}=\frac{\boldsymbol{k} \times \boldsymbol{n}}{|\boldsymbol{k} \times \boldsymbol{n}|} \quad \boldsymbol{e}^{(2)}=\boldsymbol{e}^{(3)} \times \boldsymbol{e}^{(1)} \quad \boldsymbol{e}^{(3)}=\frac{\boldsymbol{k}}{k}
$$

for $\boldsymbol{k} \times \boldsymbol{n} \neq 0$, and $\boldsymbol{e}^{(1)}, \boldsymbol{e}^{(2)}, \boldsymbol{e}^{(3)}$ may coincide with the fixed frame of reference, with

$\boldsymbol{e}^{(3)}=\boldsymbol{n}$ for $\boldsymbol{k} \| \boldsymbol{n}$. In the turbulence community, the local frame $\left(\boldsymbol{e}^{(1)}, \boldsymbol{e}^{(2)}\right)$ of the plane normal to the wavevector is often referred to as Craya-Herring frame. Accordingly, the divergence-free velocity field in wave-space has only two components in the Craya-Herring 
frame, or

$$
\hat{\boldsymbol{u}}(\boldsymbol{k}, t)=u^{(1)} \boldsymbol{e}^{(1)}+u^{(2)} \boldsymbol{e}^{(2)}
$$

For $\boldsymbol{k} \times \boldsymbol{n} \neq 0, u^{(1)}$ and $u^{(2)}$ are directly linked to the toroidal mode and the poloidal mode, respectively. For $\boldsymbol{k} \times \boldsymbol{n}=0$, they correspond to the VSHF mode. RDT equations can be written in the Craya-Herring frame, resulting in a reduced Green's function with only four independent components (Cambon, 1982). A similar decomposition is used in Bartello et al., 1994. Finally, the 'wave-vortex' decomposition introduced by Riley et al., 1981 in the particular context of stably stratified turbulence, is also a particular case of (A 1).

\section{Appendix B. Anisotropic description for second order statistics}

Independently of closure, the spectral tensor $\Phi_{i j}$ is not a general complex matrix, but has a number of special properties, including the fact that it is Hermitian, positivedefinite, and satisfies $\Phi_{i j} k_{j}=0$, obtained from the incompressibility condition $k_{j} \widehat{u}_{j}=0$. Taken together, these properties mean that, instead of the 18 real degrees of freedom of a general complex tensor, $\Phi_{i j}$ has only four. Indeed, using a spherical polar coordinate system in $\boldsymbol{k}$-space, or (A 3) and (A 4), the tensor takes the form

$$
\Phi=\left(\begin{array}{ccc}
e-\zeta_{r} & -\zeta_{i}+\mathrm{i} h & 0 \\
-\zeta_{i}-\mathrm{i} h & e+\zeta_{r} & 0 \\
0 & 0 & 0
\end{array}\right)
$$

(see Cambon et al., 1997 for details, in which $\zeta^{*}$ was denoted $Z$ ). The scalars $e(\boldsymbol{k}, t)$ and $h(\boldsymbol{k}, t)$ are real, and $\zeta(\boldsymbol{k}, t)=\zeta_{r}+\mathrm{i} \zeta_{i}$ is complex. The spectrum $e(\boldsymbol{k}, t)=\frac{1}{2} \Phi_{i i}$ is the energy density in $\boldsymbol{k}$-space, whereas $h(\boldsymbol{k}, t)=(-1 / 2) \mathrm{i} k_{l} \epsilon_{l i j} \Phi_{i j} / k$ is the helicity spectrum and, along with $\zeta$, is zero in the isotropic case.

Anisotropy is expressed through variation of these scalars with the direction of $\boldsymbol{k}$, as 
well as departures of $h$ and $\zeta$ from zero at a given wavenumber. Whatever spectral closure is used, the number of real unknowns may be reduced to the above four when carrying out numerical calculations, and the presentation of the results can be simplified using these variables, particularly when the flow is axisymmetric.

In tensorial form, the contributions from $e, \zeta, h$ in (B 1) are rewritten as

$$
\Phi_{i j}=e(\boldsymbol{k}, t) P_{i j}(\boldsymbol{k})+\Re\left[\zeta^{*}(\boldsymbol{k}, t) N_{i}(\boldsymbol{k}) N_{j}(\boldsymbol{k})\right]+\mathrm{i} h(\boldsymbol{k}, t) \epsilon_{i j n} \frac{k_{n}}{k}
$$

in which $P_{i j}=\delta_{i j}-k_{i} k_{j} / k^{2}$ is the projector and $\boldsymbol{N}=\boldsymbol{e}^{(2)}-\mathrm{i} \boldsymbol{e}^{(1)}$ using (A 3). The anisotropic contributions are separated from the isotropic one in (B 2) as

$$
\Re\left(\Phi_{i j}\right)=\underbrace{\frac{E(k)}{4 \pi k^{2}} P_{i j}}_{\text {isotropic }}+\underbrace{\left(e(\mathbf{k})-\frac{E(k)}{4 \pi k^{2}}\right) P_{i j}}_{\text {directional anisotropy }}+\underbrace{\Re\left(\zeta^{*}(\mathbf{k}, t) N_{i} N_{j}\right)}_{\text {polarization anisotropy }}
$$

with a subsequent splitting of any second-order statistical quantity.

\section{Appendix C. Integration in the vicinity of resonant surfaces}

In the limit $d=\mu_{k p q} \rightarrow 0$, the integrand of (3.3)-likewise for (3.4)-comprises a regular and a singular contributions which can be transformed into surface integrals with some additional assumptions.

Considering a resonant surface given by the couple $\left(s^{\prime}, s^{\prime \prime}\right)$, the integral is mostly contributed to by wavevectors $\boldsymbol{k}$ which are close to the resonant surface, since the integrand behaves as a Dirac function. One can therefore neglect the far wavevectors contribution, and focus mostly on a small volume $V_{\varepsilon}$ around the resonant surface $F_{s^{\prime} s^{\prime \prime}}=0$, with thickness $2 \varepsilon$. Figure 1 is a sketch of a piece of the resonant surface, and of the local system of coordinates. We introduce the local curvilinear coordinates $\left(q_{1}, q_{2}\right)$ over the resonant surface, and complete it with a third coordinate $\xi$, which represents the distance between

a point in space close to the surface to the closest one on the resonant surface. Positive values of $\xi$ are along increasing $F$, and $\xi$ is zero at the surface. Each wavevector is as- 
sociated with a point in space, so that $\boldsymbol{p}$ spans the volume around the resonant surface, and $\boldsymbol{k}^{\prime}$ is the vector associated with the closest point on the surface, consistently with the definition of $\xi$.

In the integrals (3.3) and (3.4), the non singular terms $g_{s^{\prime} s^{\prime \prime}}(\boldsymbol{p})$ and $A_{s^{\prime} s^{\prime \prime}}(\boldsymbol{p})$ are slowly varying functions in the neighbourhood of the resonant surface, with respect to the variations of $d$. They are therefore equated to their values at $\boldsymbol{k}^{\prime}$. On the contrary, $F_{s^{\prime} s^{\prime \prime}}$ is expanded in the direction orthogonal to the resonant surface, in order to remove the singularity. The first-order Taylor expansion around $\boldsymbol{k}^{\prime}$ is

$$
F(\boldsymbol{p})=F\left(\boldsymbol{k}^{\prime}+\boldsymbol{\Xi}\right)=F\left(\boldsymbol{k}^{\prime}\right)+\boldsymbol{\Xi} \cdot \boldsymbol{\nabla} F\left(\boldsymbol{k}^{\prime}\right) .
$$

Higher order terms correspond to higher order resonance, and are neglected. Since $F\left(\boldsymbol{k}^{\prime}\right)=$ 0 and the gradient of $F$ is parallel to $\boldsymbol{\Xi}, \boldsymbol{\Xi} \cdot \boldsymbol{\nabla} F\left(\boldsymbol{k}^{\prime}\right)=\xi\left|\boldsymbol{\nabla} F\left(\boldsymbol{k}^{\prime}\right)\right|$ and (C 1) becomes $F(\boldsymbol{p})=\xi\left|\nabla F\left(\boldsymbol{k}^{\prime}\right)\right|$. Using the inertial waves group velocity $\boldsymbol{C}$, another expression of the gradient is $\boldsymbol{\nabla} F\left(\boldsymbol{k}^{\prime}\right)=s^{\prime} \boldsymbol{C}\left(\boldsymbol{k}^{\prime}\right)-s^{\prime \prime} \boldsymbol{C}\left(-\boldsymbol{k}-\boldsymbol{k}^{\prime}\right)$, and yields $\left|\boldsymbol{\nabla} F\left(\boldsymbol{k}^{\prime}\right)\right|=\pi \alpha_{s^{\prime} s^{\prime \prime}}\left(\boldsymbol{k}^{\prime},-\boldsymbol{k}-\boldsymbol{k}^{\prime}\right)$, with $\alpha_{s^{\prime} s^{\prime \prime}}\left(\boldsymbol{k}^{\prime}, \boldsymbol{k}^{\prime \prime}\right)=\left|s^{\prime} \boldsymbol{C}\left(\boldsymbol{k}^{\prime}\right)-s^{\prime \prime} \boldsymbol{C}\left(\boldsymbol{k}^{\prime \prime}\right)\right| / \pi$. Finally, we obtain

$$
F(\boldsymbol{p})=\pi \alpha_{s^{\prime} s^{\prime \prime}}\left(\boldsymbol{k}^{\prime},-\boldsymbol{k}-\boldsymbol{k}^{\prime}\right) \xi
$$

which is introduced in the volume integrals with the $\left(\mathrm{d} \xi, \mathrm{d} q_{1}, \mathrm{~d} q_{2}\right)$ parameterization of the volume element. For (3.3) written in the coordinates $\left(q_{1}, q_{2}, \xi\right)$ with the element of surface $\mathrm{d} S$ from $\mathrm{d} q_{1}$ et $\mathrm{d} q_{2}$ this yields

$\int_{\mathbb{R}^{3}} g(\boldsymbol{p}) \frac{d}{d^{2}+F(\boldsymbol{p})^{2}} A_{s s^{\prime}}(\boldsymbol{p}, t) \mathrm{d}^{3} \boldsymbol{p}=\iint\left[\int \frac{d}{d^{2}+\pi^{2} \alpha_{s^{\prime} s^{\prime \prime}}^{2}\left(\boldsymbol{k}^{\prime},-\boldsymbol{k}-\boldsymbol{k}^{\prime}\right) \xi^{2}} \mathrm{~d} \xi\right] g\left(\boldsymbol{k}^{\prime}\right) A_{s s^{\prime}}\left(\boldsymbol{k}^{\prime}, t\right) \mathrm{d} S$,

where the $\xi$ integration is performed through the resonant surface. The corresponding integrand goes to zero as $\xi^{-2}$ when $\xi \rightarrow \infty$, so that the integration is extended from $-\infty$ to $\infty$ rather than a neighbourhood of the resonant surface, and can be performed 
analytically:

$$
\int_{-\infty}^{+\infty} \frac{d}{d^{2}+\pi^{2} \alpha^{2} \xi^{2}} \mathrm{~d} \xi=\frac{1}{\alpha}
$$

We thus finally get surface integrals as the asymptotic approximations for the coefficients

$$
\begin{gathered}
B_{s}(\boldsymbol{k}, t)=-2 \sum_{s^{\prime}, s^{\prime \prime}} \int_{S_{s^{\prime} s^{\prime \prime}}} \frac{g_{s^{\prime} s^{\prime \prime}}}{\alpha_{s^{\prime} s^{\prime \prime}}} A_{s s^{\prime}}\left(\boldsymbol{k}^{\prime}, t\right) \mathrm{d} S \\
=-2 \pi \sum_{s^{\prime}, s^{\prime \prime}} \int_{\mathbb{R}^{3}} \delta\left(F_{s^{\prime} s^{\prime \prime}}\right) g_{s^{\prime} s^{\prime \prime}} A_{s s^{\prime}}(\boldsymbol{p}, t) \mathrm{d}^{3} \boldsymbol{p} \\
C_{s}(\boldsymbol{k}, t)=2 \sum_{s^{\prime} s^{\prime \prime}} \int_{S_{s^{\prime} s^{\prime \prime}}} \frac{\gamma_{s^{\prime} s^{\prime \prime}}}{\alpha_{s^{\prime} s^{\prime \prime}}} A_{s s^{\prime}}\left(\boldsymbol{k}^{\prime}, t\right) A_{s s^{\prime \prime}}\left(\boldsymbol{k}^{\prime \prime}, t\right) \mathrm{d} S \\
=2 \pi \sum_{s^{\prime} s^{\prime \prime}} \int_{\mathbb{R}^{3}} \delta\left(F_{s^{\prime} s^{\prime \prime}}\right) \gamma_{s^{\prime} s^{\prime \prime}} A_{s s^{\prime}}(\boldsymbol{p}, t) A_{s s^{\prime \prime}}(\boldsymbol{q}, t) \mathrm{d}^{3} \boldsymbol{p}
\end{gathered}
$$

where $S_{s^{\prime} s^{\prime \prime}}$ is the resonant surface for the choice of parameters $\left(\boldsymbol{k}, s^{\prime}, s^{\prime \prime}\right)$ (see equation 3.6). Expressions $(\mathrm{C} 2 b)$ and $(\mathrm{C} 3 b)$ come from (3.3) and (3.4) in which the functions involving $\mu_{k p q}$ have been replaced by Dirac functions of the resonant surface, multiplied by $\pi$. Regarding $D(\boldsymbol{k}, t)$, the same procedure is applied to its real part so that

$$
D(\boldsymbol{k}, t)=-\sum_{s^{\prime}, s^{\prime \prime}} \int_{S_{s^{\prime} s^{\prime \prime}}} \frac{g_{s^{\prime} s^{\prime \prime}}}{\alpha_{s^{\prime} s^{\prime \prime}}} e\left(\boldsymbol{k}^{\prime}, t\right) \mathrm{d} S-\mathrm{i} \sum_{s^{\prime}, s^{\prime \prime}} \int_{\mathbb{R}^{3}} g_{s^{\prime} s^{\prime \prime}} \frac{F_{s^{\prime} s^{\prime \prime}}}{d^{2}+F_{s^{\prime} s^{\prime \prime}}{ }^{2}} e(\boldsymbol{p}, t) \mathrm{d}^{3} \boldsymbol{p} .
$$

The imaginary part has to be treated differently, since the singularity cannot be removed by merely letting $d$ go to zero. One has to examine the separate contributions of domains far from the resonant surface, and in its neighborhood $V_{\varepsilon}$ :

$\Im[D(\boldsymbol{k}, t)]=-\sum_{s^{\prime}, s^{\prime \prime}} \int_{\mathbb{R}^{3} \backslash V_{\varepsilon}} g_{s^{\prime} s^{\prime \prime}} \frac{F_{s^{\prime} s^{\prime \prime}}}{d^{2}+F_{s^{\prime} s^{\prime \prime}}^{2}} e(\boldsymbol{p}, t) \mathrm{d}^{3} \boldsymbol{p}-\sum_{s^{\prime}, s^{\prime \prime}} \int_{V_{\varepsilon}} g_{s^{\prime} s^{\prime \prime}} \frac{F_{s^{\prime} s^{\prime \prime}}}{d^{2}+F_{s^{\prime} s^{\prime \prime}}{ }^{2}} e(\boldsymbol{p}, t) \mathrm{d}^{3} \boldsymbol{p}$.

Away from the resonant surface, i.e. removing $V_{\varepsilon}$ from the integration domain, given $\varepsilon$, the asymptotic limit yields

$$
\lim _{d \rightarrow 0}\left[-\sum_{s^{\prime}, s^{\prime \prime}} \int_{\mathbb{R}^{3} \backslash V_{\varepsilon}} g_{s^{\prime} s^{\prime \prime}} \frac{F_{s^{\prime} s^{\prime \prime}}}{d^{2}+F_{s^{\prime} s^{\prime \prime}}^{2}} e(\boldsymbol{p}, t) \mathrm{d}^{3} \boldsymbol{p}\right]=-\sum_{s^{\prime}, s^{\prime \prime}} \int_{\mathbb{R}^{3} \backslash V_{\varepsilon}} \frac{g_{s^{\prime} s^{\prime \prime}}}{F_{s^{\prime} s^{\prime \prime}}} e(\boldsymbol{p}, t) \mathrm{d}^{3} \boldsymbol{p},
$$

Letting $\varepsilon$ go to zero, this integral tends to the principal value integral over the entire 
domain $\mathbb{R}^{3}$ denoted as

$$
\lim _{\varepsilon \rightarrow 0}\left[-\sum_{s^{\prime}, s^{\prime \prime}} \int_{\mathbb{R}^{3} \backslash V_{\varepsilon}} \frac{g_{s^{\prime} s^{\prime \prime}}}{F_{s^{\prime} s^{\prime \prime}}} e(\boldsymbol{p}, t) \mathrm{d}^{3} \boldsymbol{p}\right]=-\sum_{s^{\prime}, s^{\prime \prime}} f_{\mathbb{R}^{3}} \frac{g_{s^{\prime} s^{\prime \prime}}}{F_{s^{\prime} s^{\prime \prime}}} e(\boldsymbol{p}, t) \mathrm{d}^{3} \boldsymbol{p} .
$$

We now consider the integral over $V_{\varepsilon}$ in $(\mathrm{C} 4)$, when $d \rightarrow 0$. In the curvilinear coordinates $\left(q_{1}, q_{2}, F\right)$ the elementary volume is $\mathrm{d}^{3} \boldsymbol{p}=J\left(q_{1}, q_{2}, F\right) \mathrm{d} q_{1} \mathrm{~d} q_{2} \mathrm{~d} F$, where $J\left(q_{1}, q_{2}, F\right)$ is the Jacobian of the coordinate transformation. We assume that throughout $V_{\varepsilon}$ the functions are smooth enough to admit a Taylor expansion of the form

$$
g_{s^{\prime} s^{\prime \prime}}\left(q_{1}, q_{2}, F\right) e\left(q_{1}, q_{2}, F\right) J\left(q_{1}, q_{2}, F\right)=\sum_{i=0}^{+\infty} \alpha_{i}\left(q_{1}, q_{2}\right) F^{i}
$$

so that for the second term of (C 4) becomes

$$
\sum_{s^{\prime}, s^{\prime \prime}} \int \sum_{i=0}^{+\infty} \alpha_{i}\left(q_{1}, q_{2}\right)\left[\int_{F_{-}}^{F_{+}} \frac{F^{i+1}}{F^{2}+d^{2}} \mathrm{~d} F\right] \mathrm{d} q_{1} \mathrm{~d} q_{2},
$$

The limits $F_{+}$et $F_{-}$are functions of $q_{1}, q_{2}$ such that $F_{+}\left(q_{1}, q_{2}\right)$ and $F_{-}\left(q_{1}, q_{2}\right)$ are the values of $F$ respectively at $\left(q_{1}, q_{2}, \varepsilon\right)$ and $\left(q_{1}, q_{2},-\varepsilon\right)$ in the local coordinates system $\left(q_{1}, q_{2}, \xi\right)$.

If $i \geq 1$ the fraction in $F$ is non singular at vanishing $d$ so that for this limit:

$$
\int_{F_{-}}^{F_{+}} F^{i-1} \mathrm{~d} F=\left[\frac{F^{i}}{i}\right]_{F_{-}}^{F_{+}}=\frac{{F_{+}}^{i}-F_{-}^{i}}{i} .
$$

When $i=0$ the integral is computed as

$$
\int_{F_{-}}^{F_{+}} \frac{F}{F^{2}+d^{2}} \mathrm{~d} F=\frac{1}{2}\left[\log \left|F^{2}+d^{2}\right|\right]_{F_{-}}^{F_{+}}=\frac{1}{2} \log \left|\frac{F_{+}{ }^{2}+d^{2}}{F_{-}{ }^{2}+d^{2}}\right|
$$

prior to taking the limit $d \rightarrow 0$ :

$$
\int_{F_{-}}^{F_{+}} \frac{F}{F^{2}+d^{2}} \mathrm{~d} F \rightarrow \log \left|\frac{F_{+}}{F_{-}}\right|
$$

Equation (C 6) then becomes

$$
\sum_{s^{\prime}, s^{\prime \prime}} \int\left[\sum_{i=1}^{+\infty} \alpha_{i}\left(q_{1}, q_{2}\right) \frac{F_{+}{ }^{i}-F_{-}{ }^{i}}{i}+\alpha_{0}\left(q_{1}, q_{2}\right) \log \left|\frac{F_{+}}{F_{-}}\right|\right] \mathrm{d} q_{1} \mathrm{~d} q_{2} .
$$

Since $F$ is zero on the surface, it admits a power series expansion in $\xi$ in the form of $F\left(q_{1}, q_{2}, \xi\right)=\sum_{i=1}^{+\infty} F^{(i)}\left(q_{1}, q_{2}\right) \xi^{i}$, whence $F_{-}\left(q_{1}, q_{2}\right)=\sum_{i=1}^{+\infty} F^{(i)}\left(q_{1}, q_{2}\right)(-\varepsilon)^{i}$ and 
$F_{+}\left(q_{1}, q_{2}\right)=\sum_{i=1}^{+\infty} F^{(i)}\left(q_{1}, q_{2}\right) \varepsilon^{i}$. This is used when taking the limit $\varepsilon \rightarrow 0$ to show that $F_{-}$and $F_{+} \rightarrow 0$ and $\left|F_{+} / F_{-}\right| \rightarrow 1$, which proves that the integral (C 8) cancels out at the limit.

The asymptotic imaginary part of $D(\boldsymbol{k}, t)$ is finally

$$
\Im[D(\boldsymbol{k}, t)]=-\sum_{s^{\prime}, s^{\prime \prime}} \int_{\mathbb{R}^{3}} \frac{g_{s^{\prime} s^{\prime \prime}}}{F_{s^{\prime} s^{\prime \prime}}} e(\boldsymbol{p}, t) \mathrm{d}^{3} \boldsymbol{p} .
$$

yielding the asymptotic $D(\boldsymbol{k}, t)$ as equation $(3.10)$.

\section{Appendix D. Derivation of the geometrical factor $g_{s^{\prime} s^{\prime \prime}}$}

As proposed by e.g. Cambon, 1982, Waleffe, 1993 or Turner, 1999, an optimal factorisation of geometric coefficients is obtained by substituting to the local frame defined from the helical modes decomposition $\left(\boldsymbol{N}(s \boldsymbol{k}), \boldsymbol{N}\left(s^{\prime} \boldsymbol{p}\right), \boldsymbol{N}\left(s^{\prime \prime} \boldsymbol{q}\right)\right)$ an alternative one $\left(\boldsymbol{W}, \boldsymbol{W}^{\prime}, \boldsymbol{W}^{\prime \prime}\right)$ having its polar axis normal to the plane of the triad rather than to the plane of rotation, such that:

$$
\boldsymbol{N}(s \boldsymbol{k})=e^{s \mathrm{i} \lambda} \underbrace{(\boldsymbol{\beta}+\mathrm{i} s \boldsymbol{\gamma})}_{\boldsymbol{W}(s)}, \quad, \boldsymbol{N}\left(s^{\prime} \boldsymbol{p}\right)=e^{s^{\prime} \mathrm{i} \lambda^{\prime}} \underbrace{\left(\boldsymbol{\beta}^{\prime}+\mathrm{i} s^{\prime} \boldsymbol{\gamma}\right)}_{\boldsymbol{W}^{\prime}\left(s^{\prime}\right)}, \quad \boldsymbol{N}\left(s^{\prime \prime} \boldsymbol{q}\right)=e^{s^{\prime \prime} \mathrm{i} \lambda^{\prime \prime}} \underbrace{\left(\boldsymbol{\beta}^{\prime \prime}+\mathrm{i} s^{\prime \prime} \boldsymbol{\gamma}\right)}_{\boldsymbol{W}^{\prime \prime}\left(s^{\prime \prime}\right)}
$$

in which $\gamma$ is the unit vector normal to the plane of the triad, whereas $\boldsymbol{\beta}, \boldsymbol{\beta}^{\prime}, \boldsymbol{\beta}^{\prime \prime}$ are unit vectors normal to respectively $\boldsymbol{k}, \boldsymbol{p}, \boldsymbol{q}$ in the plane of the triad. Accordingly, scalar products in terms of $\boldsymbol{k}, \boldsymbol{p}, \boldsymbol{q}, \boldsymbol{W}, \boldsymbol{W}^{\prime}, \boldsymbol{W}^{\prime \prime}$, only depend on the moduli $k, p$ and $q$. Starting from

$$
g_{s^{\prime} s^{\prime \prime}}=-\frac{1}{4} P_{j l m}(\boldsymbol{k}) N_{j}(\boldsymbol{k}) N_{n}(-\boldsymbol{k}) N_{l}\left(s^{\prime} \boldsymbol{p}\right) N_{k}\left(-s^{\prime} \boldsymbol{p}\right) P_{r k n}(\boldsymbol{q}) N_{m}\left(s^{\prime \prime} \boldsymbol{q}\right) N_{r}\left(-s^{\prime \prime} \boldsymbol{q}\right)
$$


one finds that terms like $e^{\mathrm{i} \lambda}$ are multiplied by their conjugate, so that only the $\boldsymbol{W}$ vectors remain. Therefore $g_{s^{\prime} s^{\prime \prime}}$ may be expressed only in terms of the $k, p$ and $q$ as:

$g_{s^{\prime} s^{\prime \prime}}(k, p, q)=\frac{1}{(4 k p q)^{2}}\left[(p+q)^{2}-k^{2}\right]\left[k^{2}-(p-q)^{2}\right]\left[s^{\prime} p-k\right]\left[s^{\prime} p-s^{\prime \prime} q\right]\left[k+s^{\prime} p+s^{\prime \prime} q\right]^{2}$

Not surprisingly, this geometric factor coincides with the one involved in $\mathrm{EDQNM}_{2-3}$ when $Z$ and $h$ are ignored, which is $g_{s^{\prime} s^{\prime \prime}}=-C_{k p q}^{2} A_{1}\left(k, s^{\prime \prime} q, s^{\prime} p\right) / 4$ with $C_{k p q}=\sin \widehat{(\boldsymbol{p}, \boldsymbol{q})} / k=$ $\sin \widehat{(\boldsymbol{k}, \boldsymbol{q})} / p=\sin \widehat{(\boldsymbol{k}, \boldsymbol{p})} / q$ and $A_{1}(k, p, q)=-(p-q)(k-q)(k+p+q)^{2}$, according to equations (A3) to (A5) in the appendix of Cambon et al., 1997, using $(p+q)^{2}-k^{2}=$ $2 p q[1+\cos \widehat{(\boldsymbol{p}, \boldsymbol{q})}]$ and $k^{2}-(p-q)^{2}=2 p q[1-\cos \widehat{(\boldsymbol{p}, \boldsymbol{q})}]$.

\section{Appendix E. Numerics and validation}

\section{E.1. Numerical method}

In equation (4.1), the time derivative is estimated with a first order forward time scheme $\left.\left(\partial_{t} e\right)(t) \simeq(e(t)+\Delta t)-e(t)\right) / \Delta t$. The viscous term is centered in time and computed as $\nu k^{2}(e(t)+e(t+\Delta t))$. The energy transfer term is estimated explicitly, i.e. at time $t$ from the known spectrum $e(t)$.

As mentioned in section 4, the spatial discretisation uses wavevectors normalized by the base wavenumber $k$, since all the triads with a given base wavenumber polar angle $\theta$ are homologous. The discrete wavenumbers are $K_{j}=\left(k_{\min } / k\right) \exp \left(j \varepsilon_{\rho}\right)$ with $j \in\left\{0, \ldots, M_{\rho}\right\}$, and the logarithmic step is computed from the limit wavenumbers: $\varepsilon_{\rho}=\log \left(k_{\max } / k_{\min }\right) / M_{\rho}$. The polar direction is discretized with $\theta_{p}=(p-1 / 2) \varepsilon_{\theta}$ where $p \in\left\{1, \ldots, M_{\theta}\right\}$ and $\varepsilon_{\theta}=\pi / M_{\theta}$, and we use the same discretisation for the azimuthal angles $\phi_{n}$ with $n \in\left\{1, \ldots, M_{\phi}\right\}$. These two angles theoretically evolve between 0 and $\pi$, 
but numerically the smallest discretised angles are $\varepsilon_{\phi} / 2$ and $\varepsilon_{\theta} / 2$, and the largest ones $\pi-\varepsilon_{\phi} / 2$ and $\pi-\varepsilon_{\theta} / 2$.

Once the discretisation is chosen with $M_{\rho}, M_{\theta}$ and $M_{\phi}$, the numerical procedure starts with selecting which grid boxes intersect the resonant surface, by computing the characteristic function $F_{s^{\prime} s^{\prime \prime}}$ on the edges of the elementary volume. A change of sign indicates that the resonant surface cuts through the elementary box, and one computes the corresponding surface contribution by assuming the resonant surface to be plane within the box. The projected area of this plane onto one side of the box is first computed, and multiplied by the cosine of the projection angle to get the actual elementary surface contribution.

In the dynamical equation (4.1), the base wavevectors $\boldsymbol{k}$ scan the discretised space defined above, and within a given $e(\boldsymbol{k})$ equation, the discretised $\boldsymbol{p}$ in the energy transfer lie on the grid points. Thus the corresponding spectral energies $e(\boldsymbol{k})$ and $e(\boldsymbol{p})$ are well defined. This is not the case for $e(\boldsymbol{q})$ which has to be interpolated over the available discretised values, by a second order interpolation scheme.

Every time $\boldsymbol{q}$ falls out of the discretised space, representing triadic interactions with very small or very large wavenumbers, i.e. mostly non local interactions, the corresponding energy is neglected.

\section{E.2. Validation procedure}

Before producing the definite results with a fixed set of computational parameters, presented in this paper, we have thoroughly investigated the dependence of our numerical scheme to changes in the parameters. We have successively adopted two points of vue.

First, using the initial analytical spectrum proposed by Orszag, 1969, with unit peak wavenumber and energy (see the first spectrum of figure 5(a)), which yields the energy density spectrum $e(\boldsymbol{k})$, we have compared the various transfer spectra $T(\boldsymbol{k})$ (right-hand 
side of equation (4.1)) obtained with different values of the numerical discretisation parameters $M_{\rho}, M_{\theta}$ and $M_{\phi}$. Starting with a given arbitrary choice $M_{\rho}=M_{\theta}=M_{\phi}=100$, each of the parameter is gradually increased independently in turn, until a comparison of the values of the two transfer spectra at coincident points shows no significant change. (The difference is computed as $\| T-T_{\text {ref }}|| /|| T_{\text {ref }}||$ with a suitable norm.) One then chooses the new value of the discretisation parameter as the reference, and a fresh batch of computations of $T(\boldsymbol{k})$ is undertaken with this reference set of parameters. This not only provides a means of evaluating the dependence of the method's accuracy onto each parameter, but also permits to choose optimal values in view of the computational cost. The influence of $k_{\min }$ and $k_{\max }$ is assessed in the same way.

Three such series of tests have allowed us to move from the initial values to $M_{\rho}=200$, $M_{\theta}=M_{\phi}=100$, then to $M_{\rho}=400, M_{\theta}=300$ and $M_{\phi}=200$, and finally to $M_{\rho}=400$, $M_{\theta}=600$ and $M_{200}$. The initial values 0.01 and 100 for the minimal and maximal wavenumbers have been changed to 0.1 and 10 in the procedure. (All the figures, and an extensive presentation of the method, are available in Bellet, 2003.)

In the mean time, the integral of each energy transfer over wavespace is computed to check how well energy conservation is verified for each run. The convergence with increasing $M_{\rho}$ is plotted on figure 2 .

The second point of vue for the study of the numerical scheme regards the time dependent spectrum. The effective Reynolds number is first chosen to be $R e=1$, with the same discretisation parameters as above, and $\Delta t=0.01$, and the computation goes to the final time $t_{f}=0.38$. The influence of the time step is studied by decreasing the initial value, and appears to be small, so this figure is kept. Then again, the influence of the spatial resolution is studied in a series of non stationary computations, with $M_{\rho}$ increased up to 800 , and $M_{\theta}$ to 900 . (The influence of $M_{\phi}$ was shown in the previous runs to be negligible 
once the chosen value 100 was reached.) In these runs, the round off errors and the radial cut off at large wavenumbers are identified to be possible sources of numerical problems in the large wavenumber range of the spectrum, showing the necessity of a well-chosen $k_{\max }$. (We have tested values of up to $k_{\max }=60$.)

For this reason, in view of the available computational resources, and in order to be able to perform the final computation as long as possible in time, we have only slightly increased the value of the effective Reynolds number to $R e=5$ to take advantage of the stabilizing role of viscosity at very small scale. The reference case whose results are presented in this work also uses $M_{\rho}=400, M_{\theta}=300, M_{\phi}=100, k_{\min }=0.1, k_{\max }=60$ and $\Delta t=5 \cdot 10^{-5}$. The run is performed from the initial time $t=0$ to $t_{f}=1.05$.

\section{REFERENCES}

Babin, A., Mahalov, A. , \& Nicolaenko, B. 1997. Regularity and Integrability of 3D Euler and Navier-Stokes Equations for Uniformly Rotating Fluids. Asympt. Anal., 15(2), $103-150$.

Babin, A., Mahalov, A. , \& Nicolaenko, B. 1999. Global Regularity of 3D Rotating NavierStokes Equations for Resonant Domains. Indiana University Mathematics Journal, 48(3), $1133-1176$.

Babin, A. , Mahalov, A. , \& Nicolaenko, B. 2001. 3D Navier-Stokes and Euler Equations with Initial Data Characterized by Uniformly Large Vorticity. Indiana University Mathematics Journal, 50, 1-35.

Bardina, J. , Ferziger, J.M. , \& Rogallo, R.S. 1985. Effect of rotation on isotropic turbulence: computation and modelling. J. Fluid Mech., 154, 321-326.

Bartello, P. , MÉtais, O. , \& Lesieur, M. 1994. Coherent structures in rotating threedimensional turbulence. J. Fluid Mech., 173, 1-29.

Bellet, FABIEn 2003. Étude asymptotique de la turbulence d'ondes en rotation. PhD thesis, École Centrale de Lyon. 
Benney, D.J. \& Newell, A.C. 1967. Sequential time closures for interacting random waves. J. Math. and Phys., 46, 363-393.

Benney, D.J. \& Saffman, P.G. 1966. Nonlinear interaction of random waves in a dispersive medium. Proc. R. Soc. London Ser. A, 289, 301-320.

Caillol, P. \& Zeitlin, V. 2000. Kinetic equations and stationary energy spectra of weakly nonlinear internal gravity waves. Dyn. Atm. Oceans, 32, 81-112.

Cambon, C. 1982. Étude spectrale d'un champ turbulent incompressible soumis à des effets couplés de déformation et de rotation imposés extérieurement. PhD thesis, Univ. Lyon I, France.

Cambon, C. , Godeferd, F. S. , Nicolleau, F.C.G.A. , \& Vassilicos, J.C. 2004a. Turbulent diffusion in rapidly rotating flows with and without stable stratification. J. Fluid Mech., 499, 231-255.

Cambon, C. \& Jacquin, L. 1989. Spectral approach to non-isotropic turbulence subjected to rotation. J. Fluid Mech, 202, 295-317.

Cambon, C. , Jacquin, L. , \& Lubrano, J.-L. 1992. Toward a New Reynolds Stress Model for Rotating Turbulence. Phys. Fluids A, 4(4), 812-824.

Cambon, C. , Mansour, N.N. , \& Godeferd, F.S. 1997. Energy transfer in rotating turbulence. J. Fluid Mech., 337, 303-332.

Cambon, C. , Rubinstein, R. , \& Godeferd, F. S. 2004b. Advances in wave turbulence: rapidly rotating flows. New J. of Phys., 6(73).

Cambon, C. \& Scott, J. F. 1999. Linear and nonlinear models of anisotropic turbulence. Annu. Rev. Fluid Mech., 31, 1-53.

Comte-Bellot, G. \& Corrsin, S. 1971. Simple eulerian time-correlation of full and narrowband velocity signals in grid-generated isotropic turbulence. J. Fluid Mech., 48, 273-337.

CRAYA, A.D.D. 1958. Contribution à l'analyse de la turbulence associée à des vitesses moyennes. P.S.T. 345, Ministère de l'air, France.

Galtier, S. 2003. A weak inertial wave turbulence theory. Phys. Rev. E, 68, 015301-1-4.

Galtier, S. , Nazarenko, S. , Newell, A. C. , \& Pouquet, A. 2000. A weak turbulence theory for incompressible MHD. J. Plasma Physics, 63, 447-488. 
Godeferd, F.S. \& Cambon, C. 1994. Detailed investigation of energy transfers in homogeneous stratified turbulence. Phys. Fluids, 6 (6), 2084-2100.

Godeferd, F.S. \& Staquet, C. 2000. Statistical modelling and direct numerical simulations of decaying stably-stratified turbulence: Part 2: Large and small scales anisotropy. J. Fluid Mech., submitted.

Godeferd, F. S. \& Lollini, L. 1999. Direct numerical simulations of turbulence with confinement and rotation. J. Fluid Mech., 393, 257-308.

Greenspan, H. P. 1990. The theory of rotating fluids. Cambridge University press (1968), Cambridge, reprinted by Breukelen Press, Brookline, MA.

Hossain, M. 1994. Reduction in the dimensionality of turbulence due to a strong rotation. Phys. Fluids, 6, 1077-1080.

Jacquin, L. , Leuchter, O. , Cambon, C. , \& Mathieu, J. 1990. Homogeneous turbulence in the presence of rotation. J. Fluid Mech., 220, 1-52.

Kraichnan, R. H. 1958. The structure of isotropic turbulence at very high Reynolds numbers. J. Fluid Mech., 5(2), 497-543.

LeIth, C. E. 1971. Atmospheric predicability and two dimensional turbulence. J. Atmos. Sci., $28,145-161$.

Lvov, Yuri \& TABAK, Esteban 2001. Hamiltonian Formalism and the Garrett-Munk Spectrum of Internal Waves in the Ocean. Phys. Rev. Lett., 87(168501).

McEwan, A. D. 1970. Inertial oscillations in a rotating fluid cylinder. J. Fluid Mech., 40(3), 603-640.

Monin, A. \& Yaglom, A. 1975. Statistical Fluid Mechanics: Mechanics of Turbulence (2 vol.). MIT Press, Cambridge, MA.

Morinishi, Y. , Nakabayashi, K. , \& Ren, S.Q. 2001a. Dynamics of anisotropy on decaying homogeneous turbulence subjected to system rotation. Phys. Fluids, 13, 2912.

Morinishi, Y. , Nakabayashi, K. , \& Ren, S.Q. 2001b. A new DNS algorithm for rotating homogeneous turbulence. Int. J. Heat and Fluid Flow, 22, 30-38.

Mowbray, D.E. \& Rarity, D.E. 1967. A theoretical and experimental investigation of the 
phase configuration of internal waves of small amplitude in a density stratified liquid. $J$. Fluid Mech., 28, 1-16.

Orszag, S.A. 1969. Numerical methods for the simulation of turbulence. Phys. of Fluids, SUPP II, 12, 250-257.

OrszaG, S.A. 1970. Analytical theories of turbulence. J. Fluid Mech., 41, 363.

Pouquet, A. , Lesieur, M. , André, J.C. , \& Basdevant, C. 1975. Evolution of high Reynolds number two-dimensional turbulence. J. Fluid Mech., pages 305+.

Reynolds, W. C. \& Kassinos, S. 1995. One-point modeling for rapidly deformed homogeneous turbulence. Proc. R. Soc. London Ser. A, 451, 87-104.

Riley, J.J , Metcalfe, R.W. , \& Weissman, M.A. 1981. Direct numerical simulations of homogeneous turbulence in density-stratified fluids. In West, B.J. , editor, Proceedings of AIP Conference on Nonlinear Properties of Internal Waves, pages 79-112, New York. American Institute of Physics.

Smith, L. \& LeE, Y. 2005. On near resonances and symmetry breaking in forced rotating flows at moderate Rossby number. J. Fluid Mech., to appear.

Smith, L. \& Waleffe, F. 1999. Transfer of energy to two-dimensional large scales in forced, rotating three-dimensionnal turbulence. Phys. Fluids, 11(6), 1608-1622.

Smith, L. \& Waleffe, F. 2002. Generation of slow large scales in forced rotating stratified turbulence. J. Fluid Mech., 451, 145-168.

Squires, K. D. , Chasnov, J. R. , Mansour, N. N. , \& Cambon, C. 1994. The asymptotic state of rotating homogeneous turbulence at high Reynolds number. In Application of Direct and Large Eddy Simulation to transition and turbulence, Chania, Crete, Greece.

Staquet, C. \& Sommeria, J. 2002. Internal gravity waves: from instabilities to turbulence. Annu. Rev. Fluid Mech, 34, 559-593.

Turner, L 1999. Macroscopic structures of inhomogeneous, Navier-Stokes turbulence. Phys. Fluids, 11, 2367-2380.

Waleffe, F. 1993. Inertial transfers in the helical decomposition. Phys. Fluid A, 5, 677-685.

Yang, X. \& Domaradzki, J.A. 2004. LES of decaying rotating turbulence. J. Phys. Fluid, to appear. 
Zakharov, V.E., Lvov, V., \& Falkovich, G. 1992. Wave turbulence. Springer-Verlag, New-York.

Zноu, Y. 1995. A phenomenological treatment of rotating turbulence. Phys. Fluids, 7, 2092. 


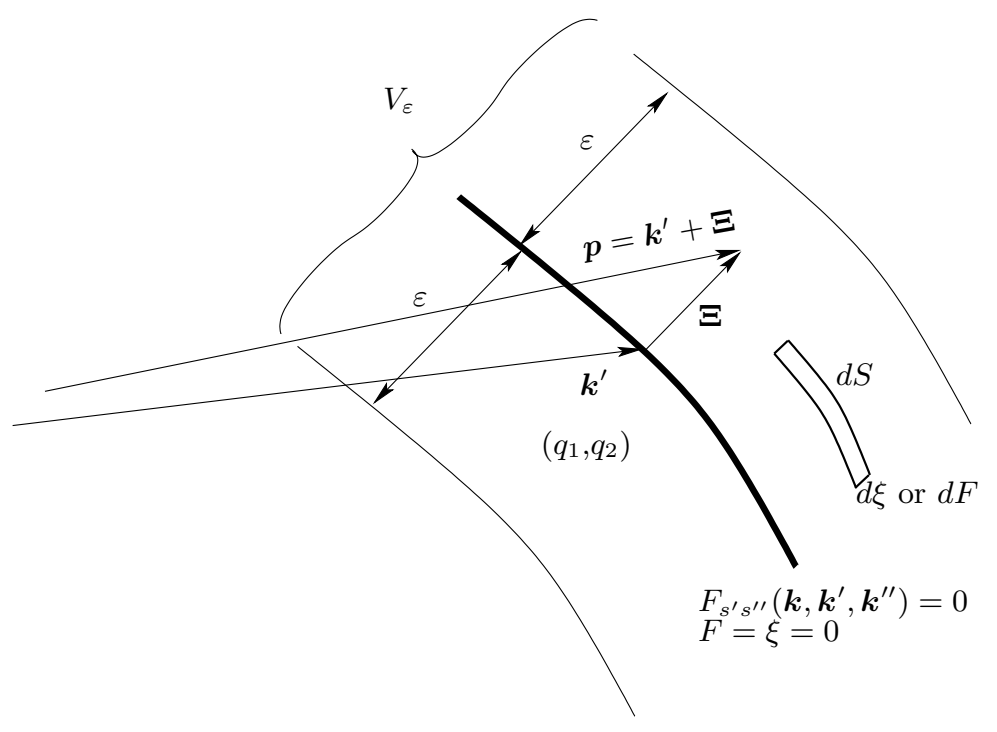

Figure 1. General curvilinear coordinate system in the neighbourhood of a resonant surface. 


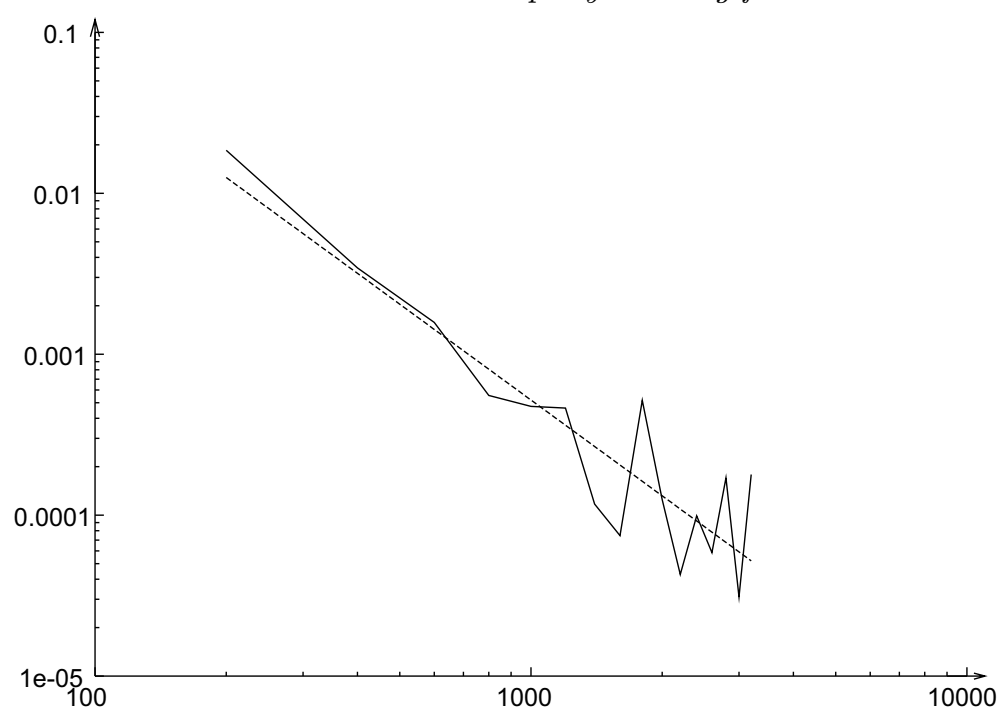

FIGURE 2. Variation of the unsigned value of the integrated transfer as a function of $M_{\rho}$, with $M_{\theta}=200, M_{\phi}=100, k_{m}=0.1$ and $k_{\max }=10$. - - - $\quad$ The least square fit with a line is also shown in dashes, exhibiting a $M_{\rho}^{-1.98}$ dependency. 


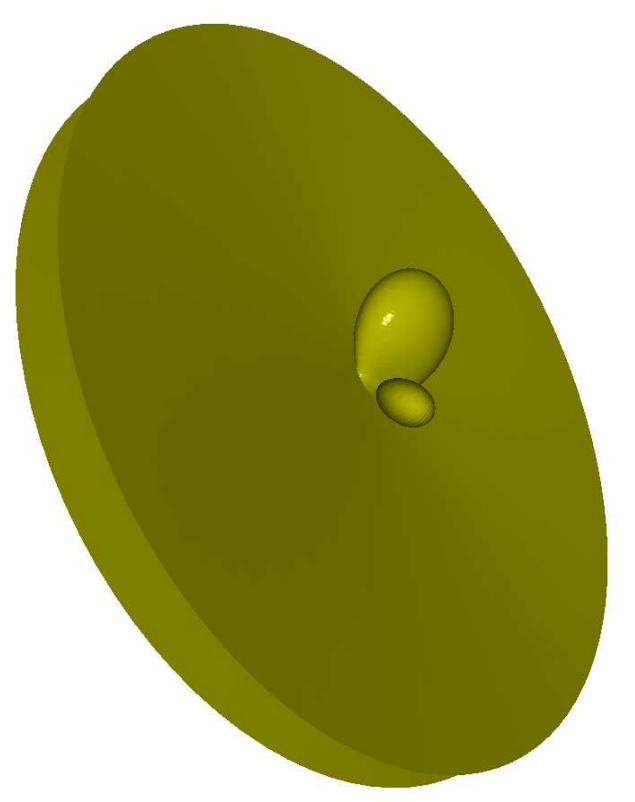

FiguRE 3. Three-dimensional rendering of the resonant surface, i.e. the locus of $\boldsymbol{p}$, at $k=1$ and $\theta_{k}=1.4$, with 100 points of discretisation for wavenumbers, latitude and azimuthal angles. The large conical-shaped fold is theoretically infinite, but is truncated at the maximum wavenumber. Two smaller closed folds are observed on top of it. The same symmetrical folds exist behind these, only hinted here. Note that the points connecting the closed folds and the infinite one are singular and quite complex to accurately account for. 


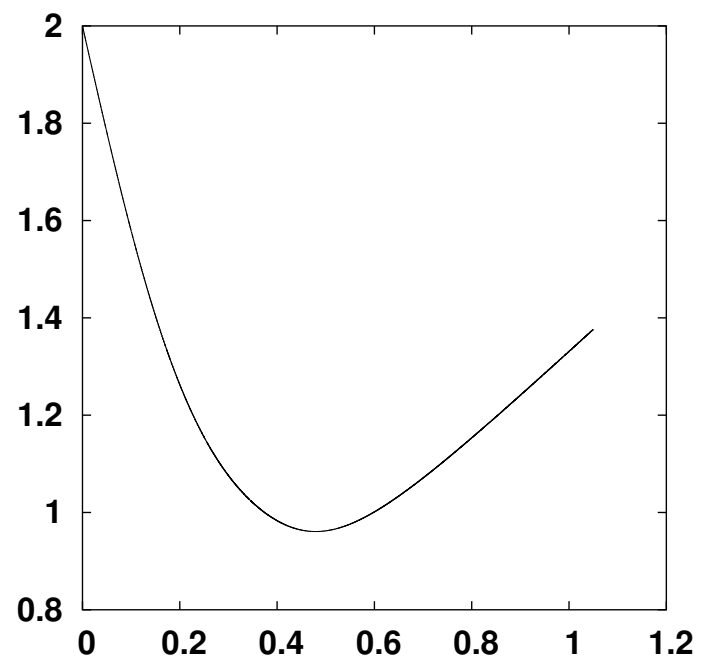

Figure 4 . Ratio $k / \epsilon$ as a function of time. 

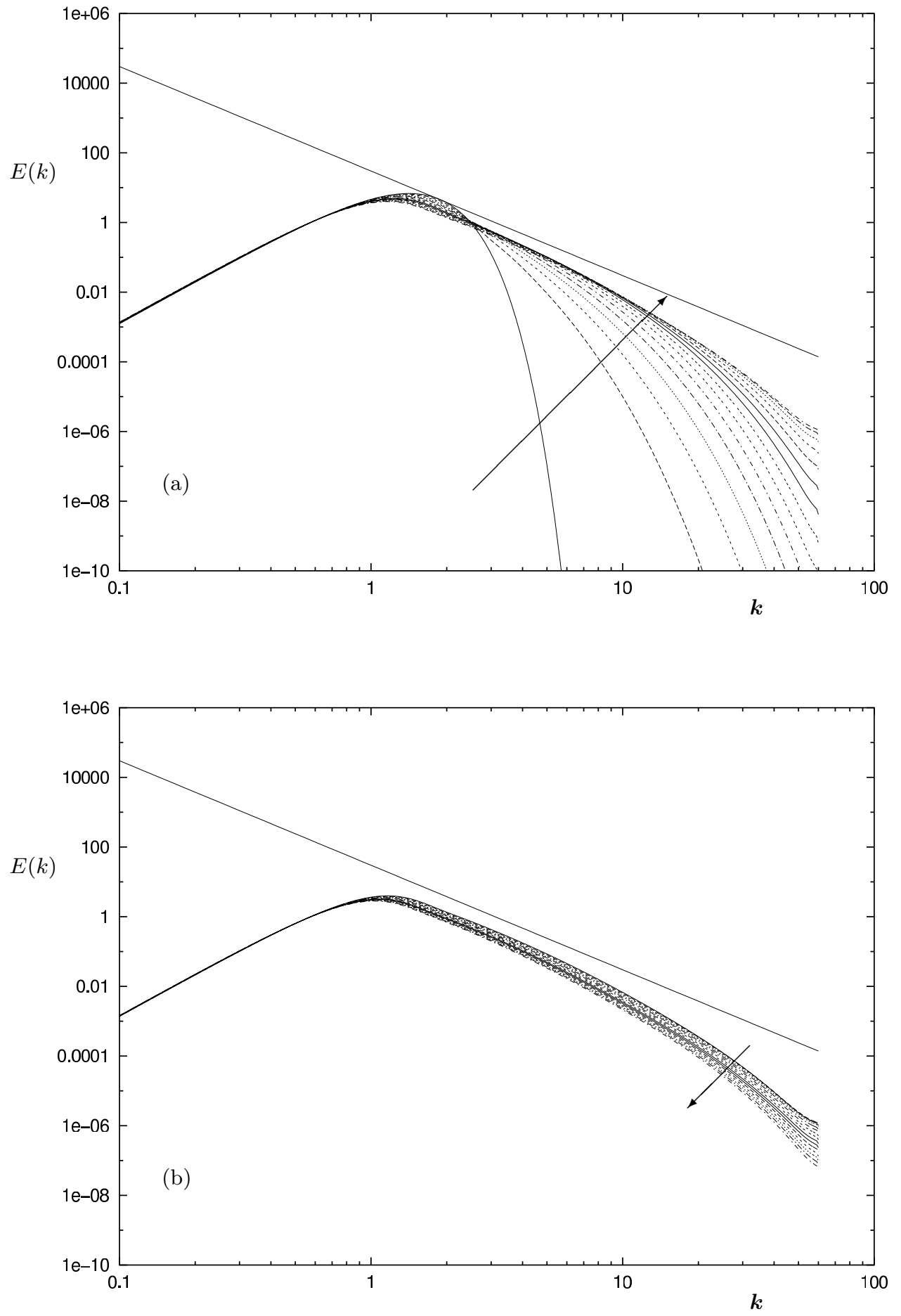

FiguRE 5. Temporal evolution of the spherically integrated energy spectrum at $R e=5$, $k_{\max }=60, d t=5 \cdot 10^{-5}$ : (a) between $t_{0}=0$ and $t=0.525$ by steps of 0.0375 ; (b) between $t=0.525$ and $t_{f}=1.05$ with the same timestep. The arrows denote increasing time and the straight line shows the $k^{-3}$ dependency. 

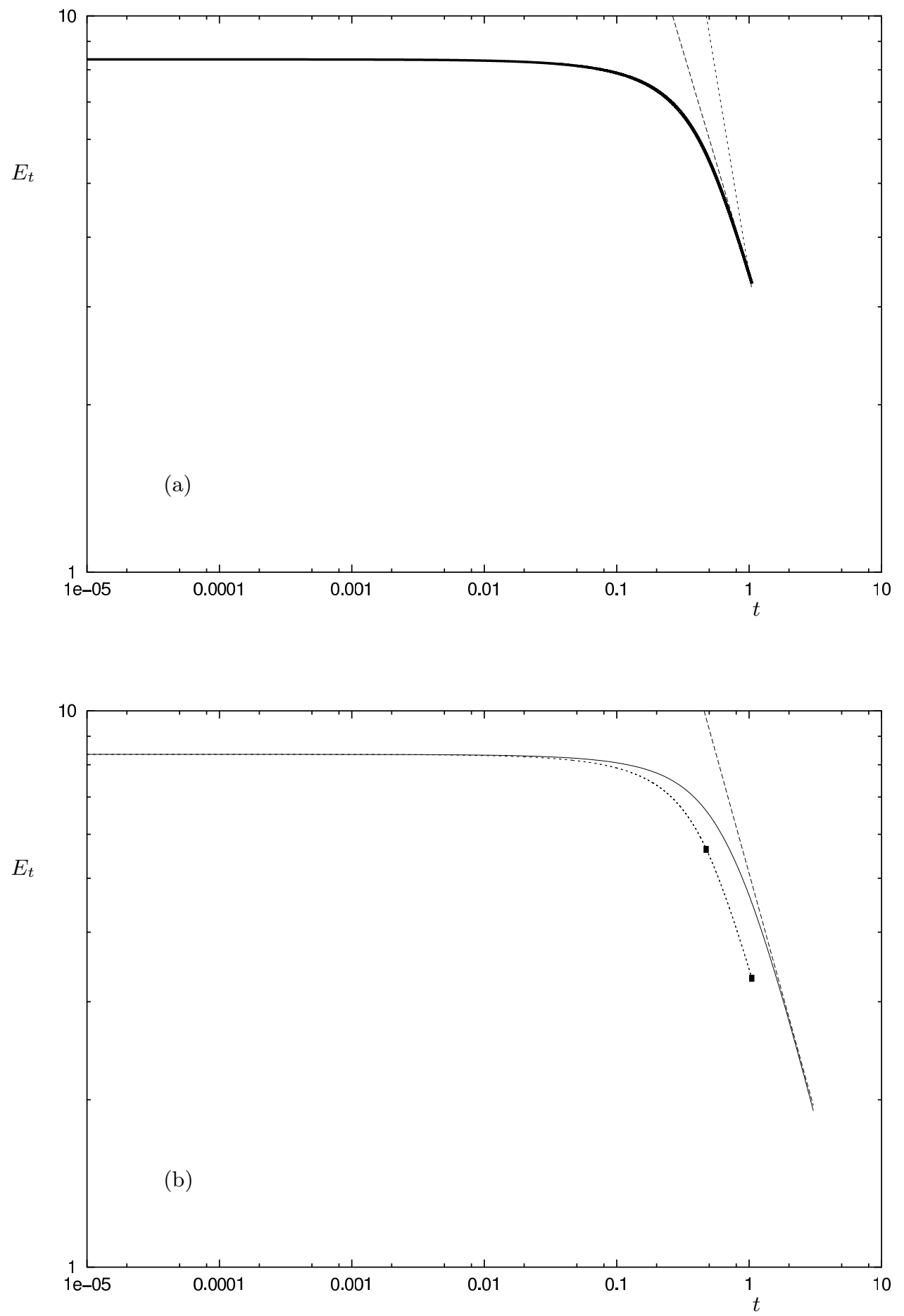

Figure 6. (a) - Time evolution between $t=0$ et $t=1.05$ with $R e=5, k_{\max }=60$ and $d t=5 \cdot 10^{-5}$ of the total energy $E_{t} .----t^{-0.8}$ and $----t^{-10 / 7}$ power laws. (b) $\mathrm{EDQNM}_{2}$ and ---- AQNM decay of total energy $E_{t}$. The straight line is a $t^{-0.86}$ law. The black squares show the energy at times $t=0.525$ and $t=1.05$, at which the angular dependent spectra are plotted on figure 8 . 

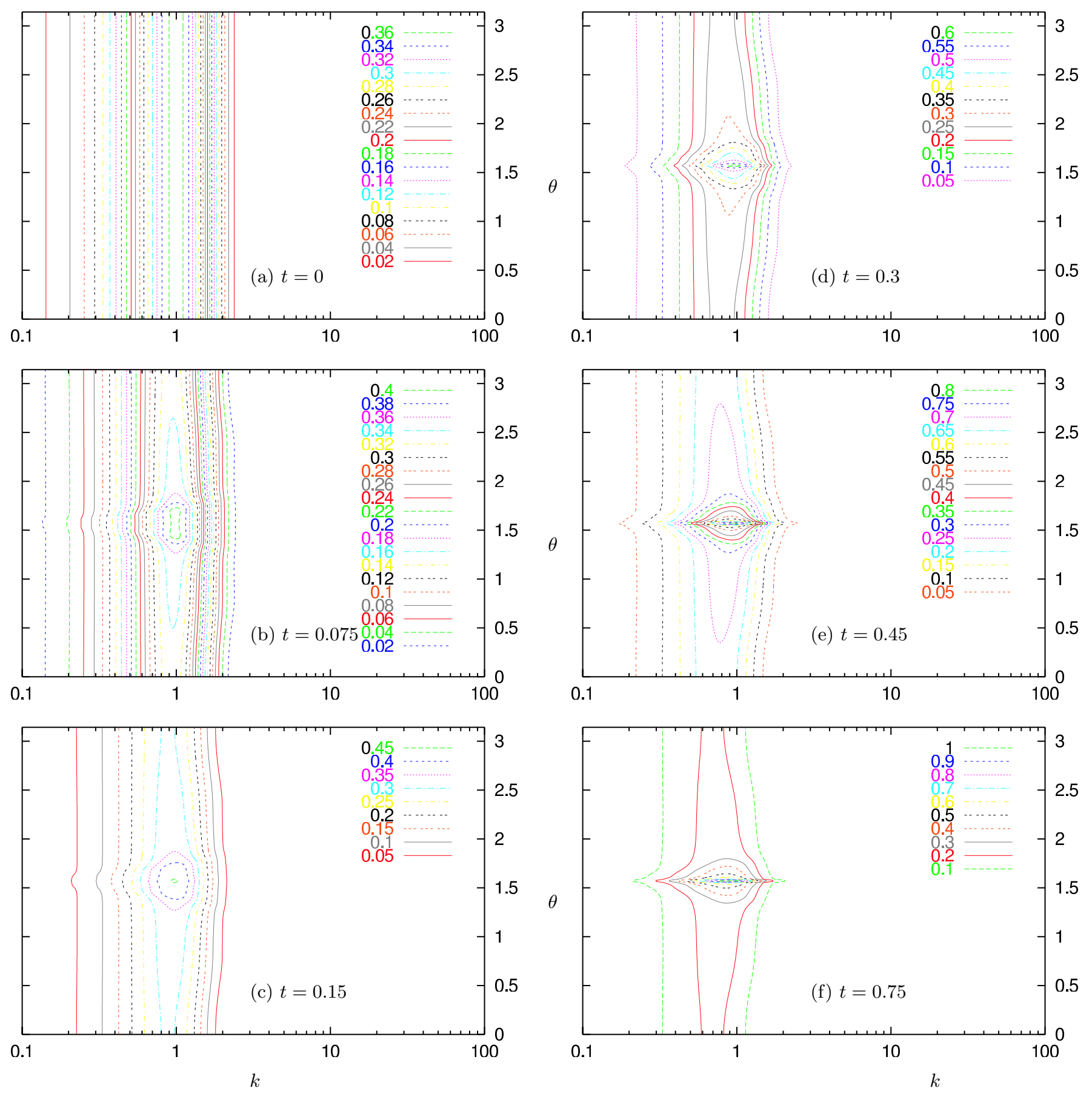

FiguRE 7. Isovalues of the spectral energy density $e(k, \theta)$ at $R e=5, k_{\max }=60, d t=5 \cdot 10^{-5}$ between $t=0$ et $t=0.75$. 

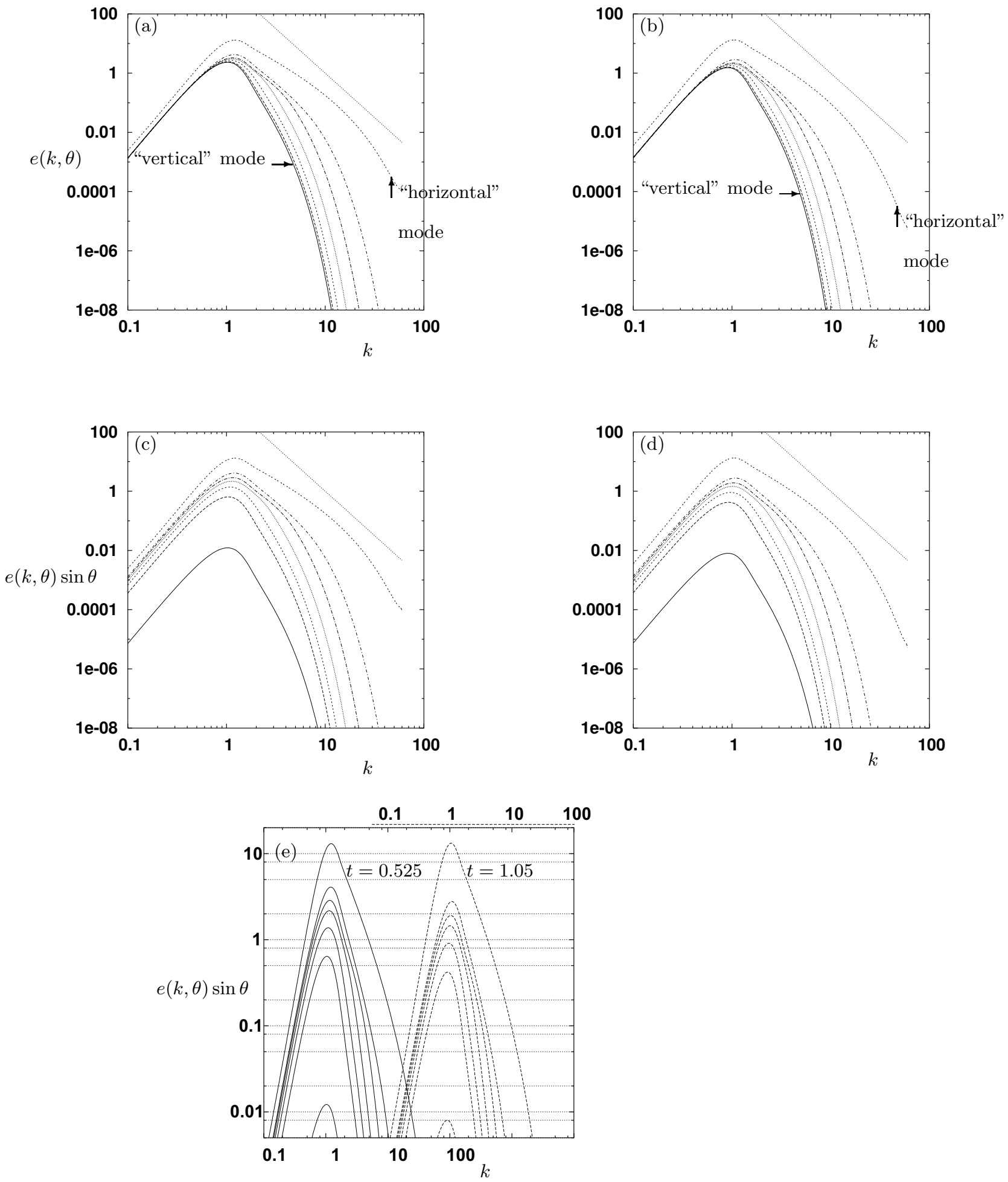

Figure 8. Spectral energy density for different angles (from bottom to top, $\theta /(\pi / 2)=1 / 300-i . e$. what we call the "vertical" mode-, 51/300, 101/300, 151/300, $201 / 300,251 / 300$ and 299/300 - the "horizontal" mode) at $R e=5, k_{\max }=60, d t=5 \cdot 10^{-5}$, at (a) $t=0.525$ and (b) $t=1.05$. (c) and (d) represent the same quantity multiplied by the corresponding $\sin \theta$ for each given angle. The $k^{-3}$ slope is also plotted. (e) Same as (c) and (d) with a zoomed scale and data at $t=1.05$ shifted two decades to the right (axis labels on top). 


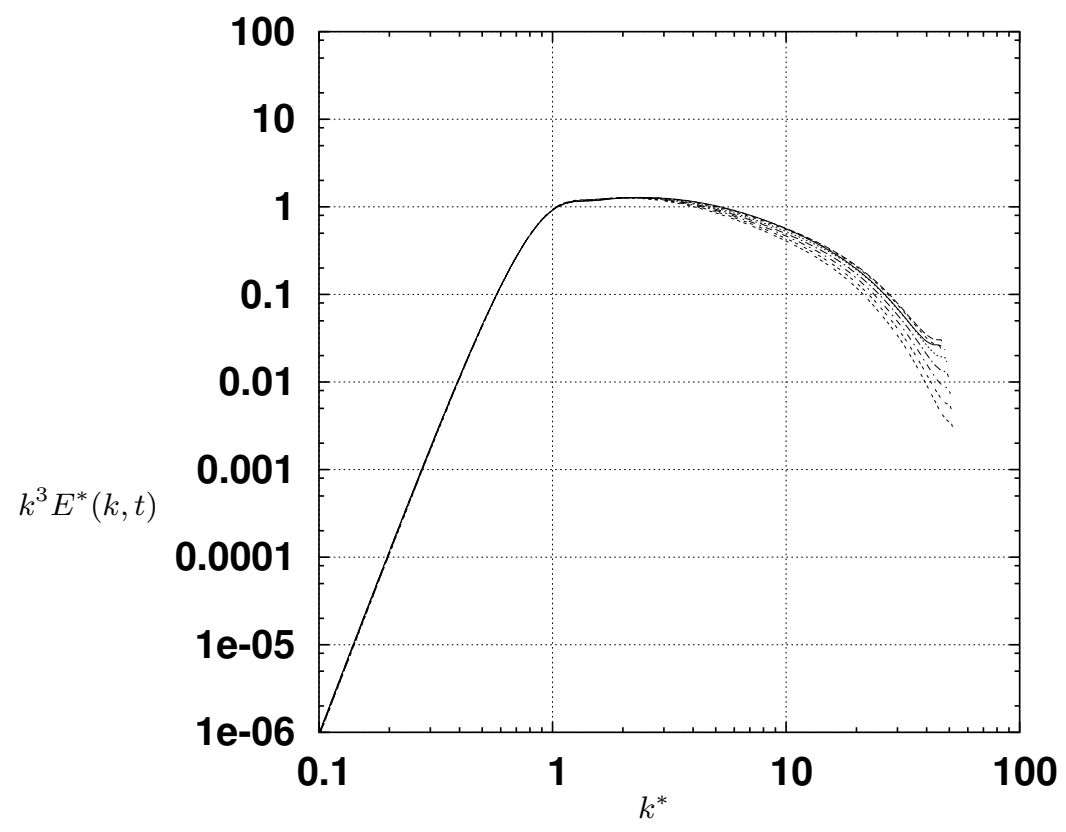

FIgURE 9. Self-similarity of the spherically integrated energy spectra multiplied by $k^{3}$ at times $t$ between 0.525 and 1.05 , renormalized by the time dependent total kinetic energy, plotted as a function of the wavenumber $k^{*}$, renormalized by the time dependent peak wavenumber. 
Wave-turbulence in rapidly rotating flows
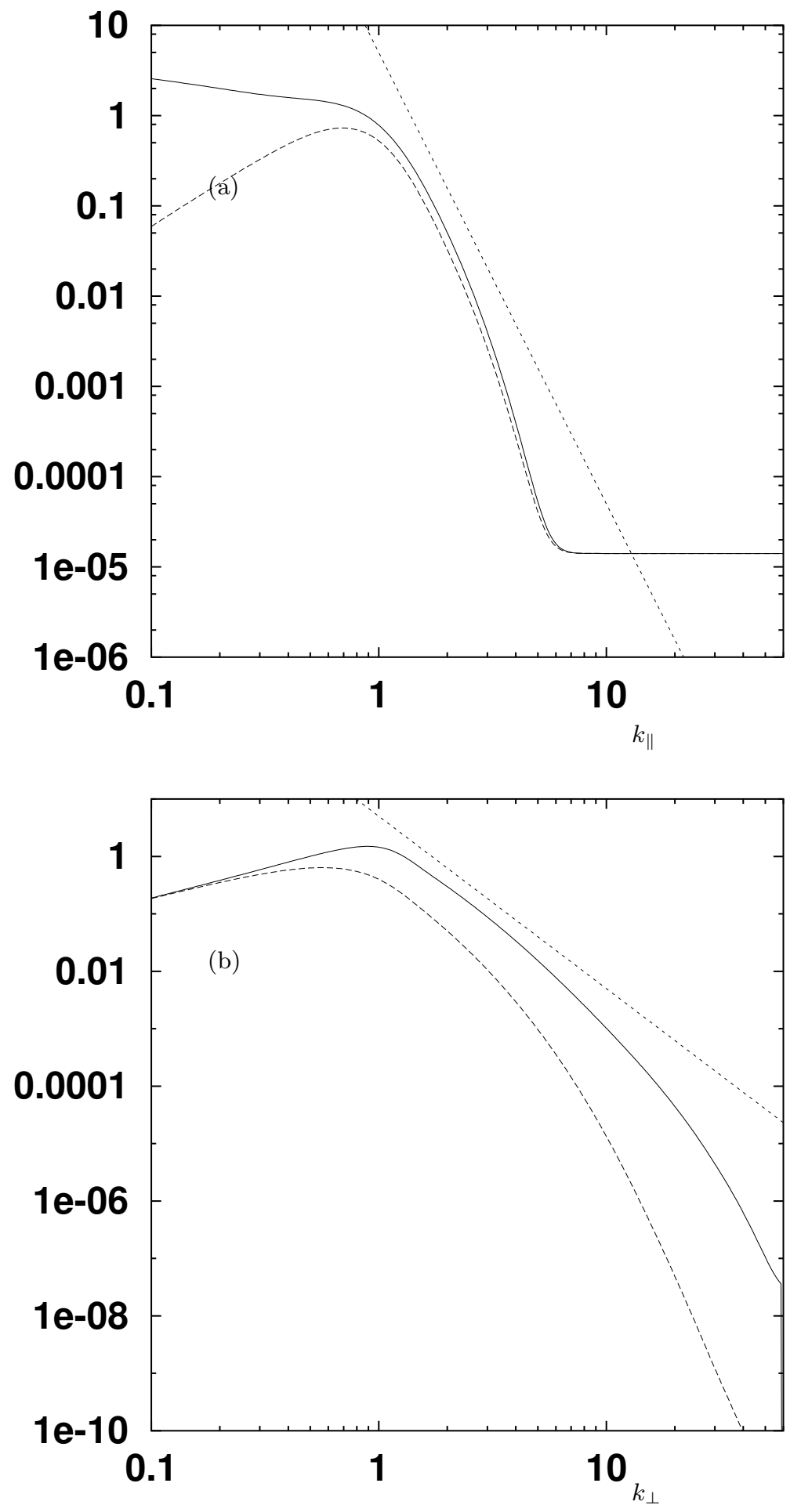

FiguRE 10. (a) One dimensional spectra $E_{h, v}\left(k_{\|}\right)$integrated on planes as a function of the vertical wavenumber component $k_{\|}$; (b) one dimensional spectra $E_{h, v}\left(k_{\perp}\right)$ integrated over cylinders as a function of the horizontal wavenumber $k_{\perp}$, at $R e=5, k_{\max }=60, d t=5 \cdot 10^{-5}$ at $t=1.05$. Solid lines show the horizontal correlation spectrum $E_{h}$, and dashed lines the vertical correlation spectrum $E_{v}$. Straight lines show: (a) $k^{-5}$ power law; (b) $k^{-3}$ power law. 

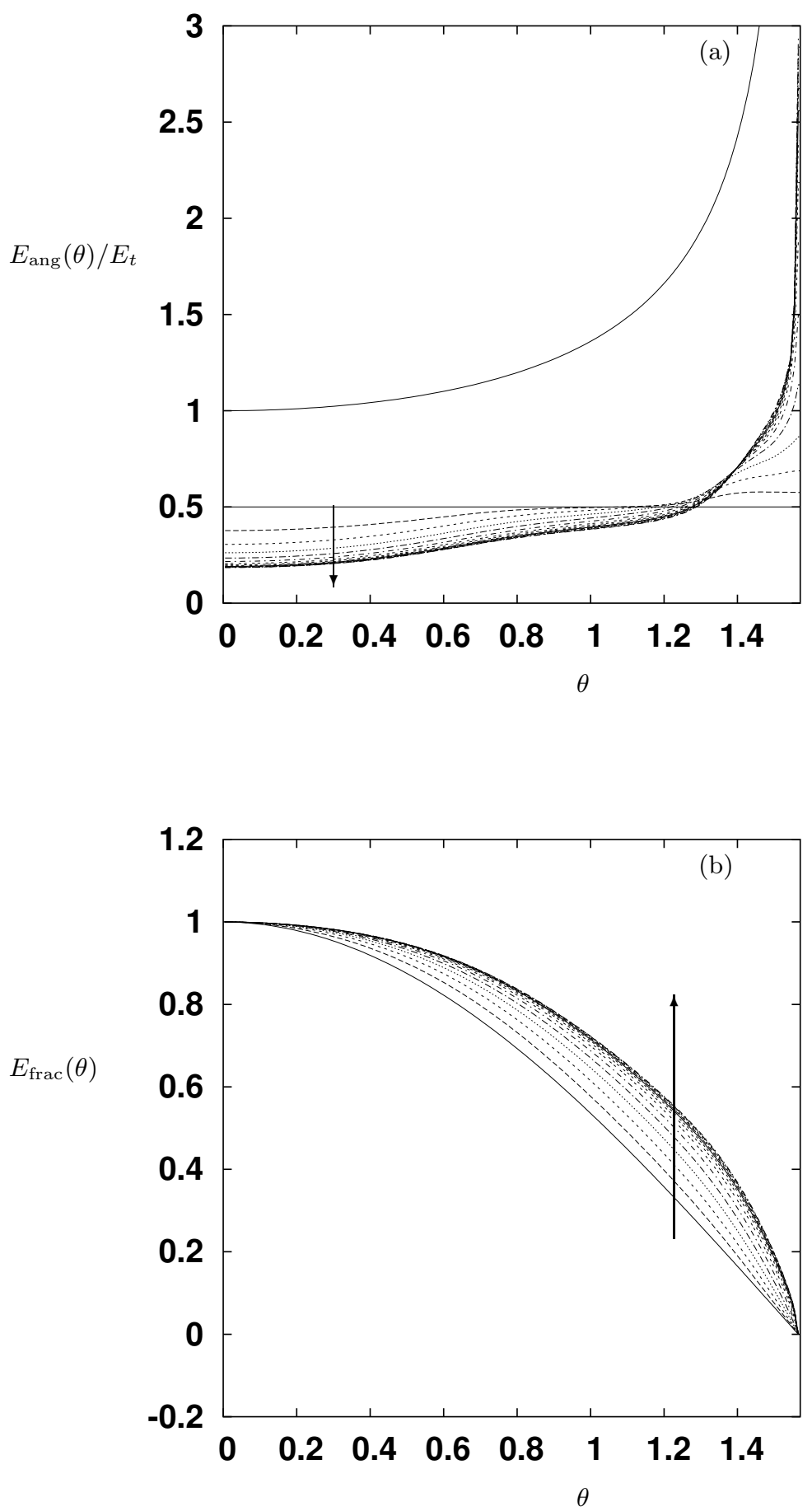

Figure 11. Time evolution of (a) $E_{\text {ang }}(\theta) / E_{t}$ at $R e=5, k_{\max }=60, d t=5 \cdot 10^{-5}$, between $t=0$ et $t=1.05$. (The upper most solid line shows a $1 / \sqrt{\cos \theta}$ dependency.) (b) $E_{\text {frac }}(\theta)$ for the same set of parameters. 

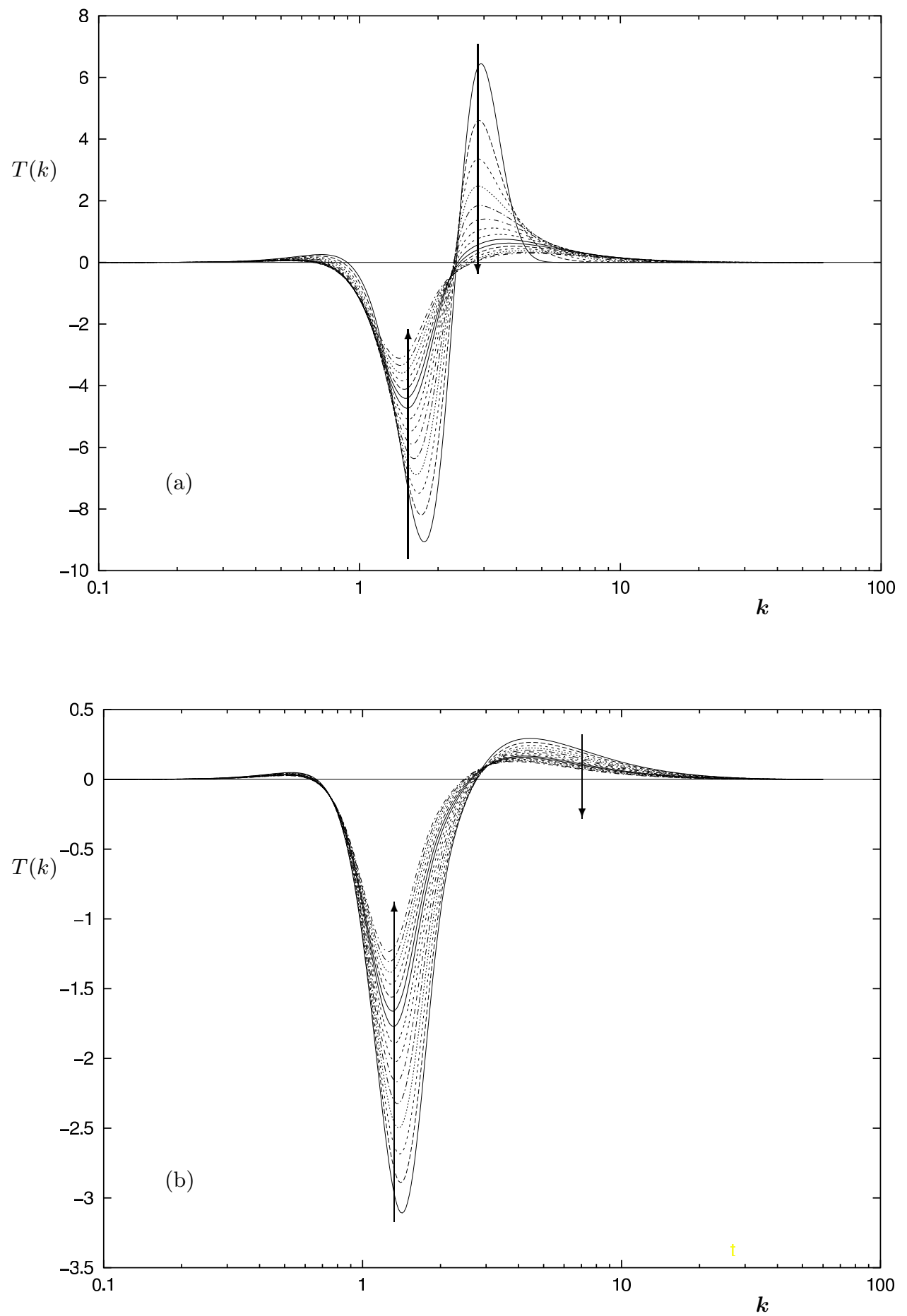

FiguRE 12. Time evolution of the spherically integrated energy transfer spectrum at $R e=5$, $k_{\max }=60, d t=5 \cdot 10^{-5}$ between (a) $t_{0}=0$ and $t=0.525$; (b) $t=0.525$ and $t=1.05$ by steps of 0.0375 . Arrows indicate increasing time. 


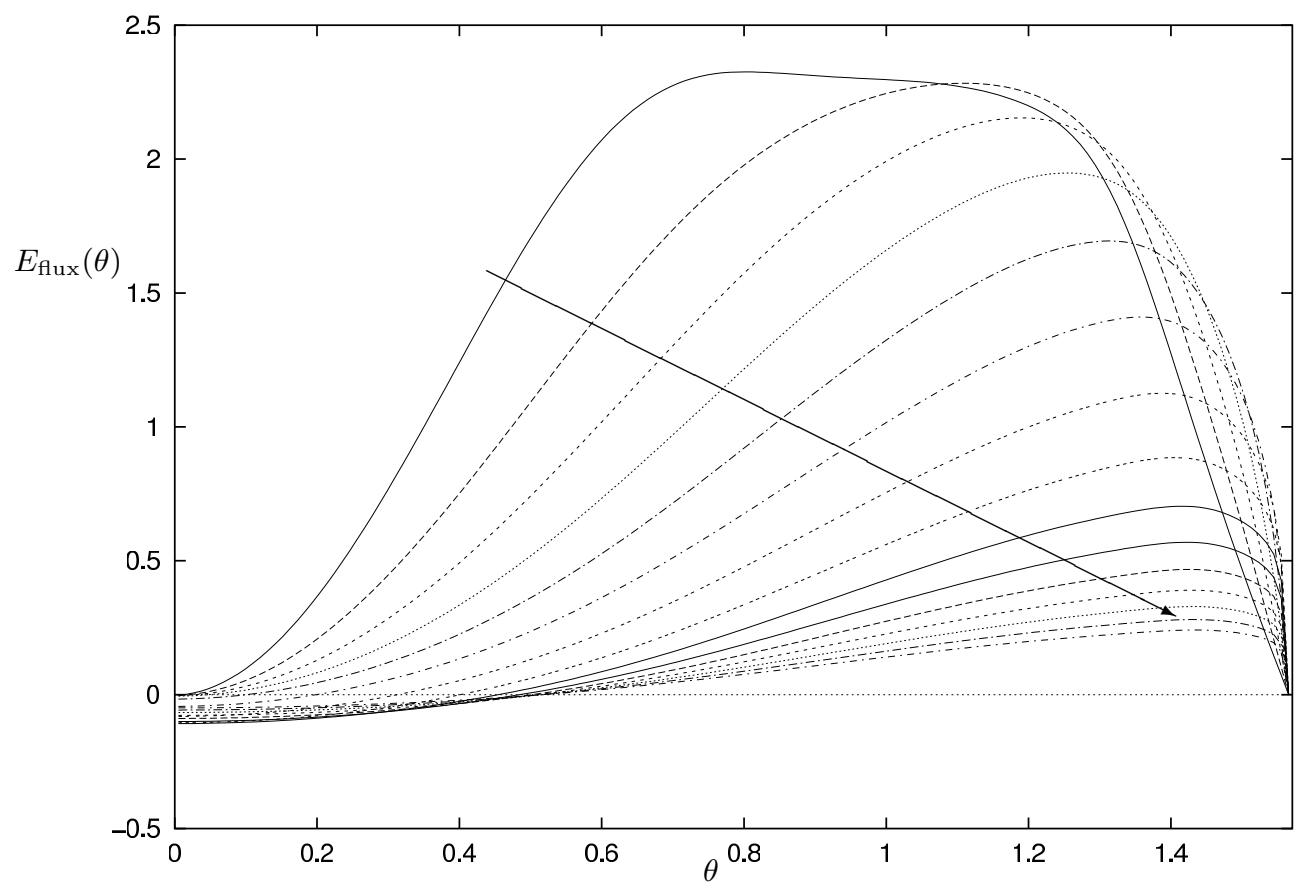

Figure 13. Time evolution of the angular flux of energy $E_{\text {flux }}(\theta)$ as a function of $\theta$, at $R e=5$,

$$
k_{\text {max }}=60, d t=5 \cdot 10^{-5} \text {, between } t=0 \text { et } t=1.05 \text {. }
$$




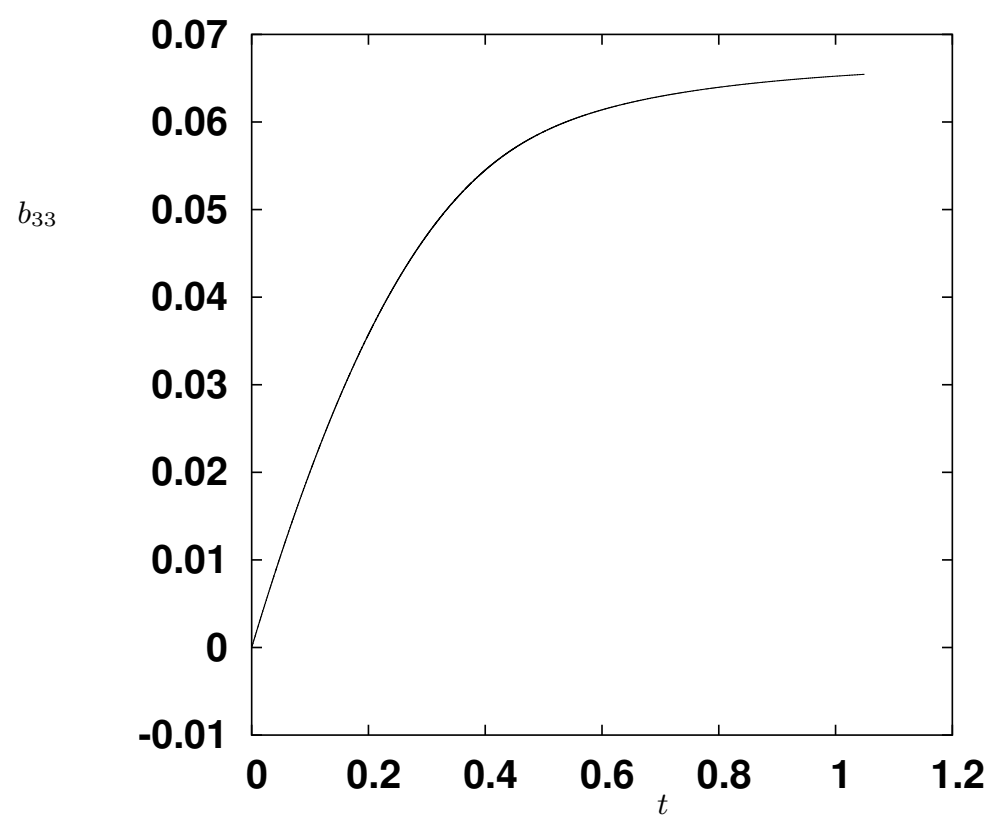

Figure 14. Time evolution of the deviatoric component of the Reynolds stress tensor $b_{33}$ at $R e=5, k_{\max }=60, d t=5 \cdot 10^{-5}$, between $t=0$ et $t=1.05$. 